\title{
Brain and spinal cord injury repair by implantation of human neural progenitor cells seeded onto polymer scaffolds
}

\author{
Jeong Eun Shin', Kwangsoo Jung², Miri Kim², Kyujin Hwang ${ }^{2}$, Haejin Lee ${ }^{3}$, Il-Sun Kim³ , Bae Hwan Lee (1) \\ II-Shin Lee ${ }^{3}$ and Kook In Park Pa, $^{1,2,3}$
}

\begin{abstract}
Hypoxic-ischemic ( $\mathrm{HI})$ brain injury and spinal cord injury ( $\mathrm{SCl}$ ) lead to extensive tissue loss and axonal degeneration. The combined application of the polymer scaffold and neural progenitor cells (NPCs) has been reported to enhance neural repair, protection and regeneration through multiple modes of action following neural injury. This study investigated the reparative ability and therapeutic potentials of biological bridges composed of human fetal brainderived NPCs seeded upon poly(glycolic acid)-based scaffold implanted into the infarction cavity of a neonatal HI brain injury or the hemisection cavity in an adult SCl. Implantation of human NPC (hNPC)-scaffold complex reduced the lesion volume, induced survival, engraftment, and differentiation of grafted cells, increased neovascularization, inhibited glial scar formation, altered the microglial/macrophage response, promoted neurite outgrowth and axonal extension within the lesion site, and facilitated the connection of damaged neural circuits. Tract tracing demonstrated that hNPC-scaffold grafts appear to reform the connections between neurons and their targets in both cerebral hemispheres in HI brain injury and protect some injured corticospinal fibers in SCl. Finally, the hNPC-scaffold complex grafts significantly improved motosensory function and attenuated neuropathic pain over that of the controls. These findings suggest that, with further investigation, this optimized multidisciplinary approach of combining hNPCs with biomaterial scaffolds provides a more versatile treatment for brain injury and SCl.
\end{abstract}

\section{Introduction}

Hypoxic-ischemic (HI) brain injury, a major cause of death and serious neurological disability among patients of all age groups, leads to vast loss of cerebral parenchyma, neural cells, and neural connections. Traumatic spinal cord injury (SCI) causes spinal cavitary lesion, loss of neurons and oligodendrocytes, axonal damage, demyelination, and glial scar formation, resulting in devastating lifelong motor/sensory dysfunctions for patients. Although extensive research is underway to

\footnotetext{
Correspondence: II-Shin Lee (sunew@yuhs.ac) or Kook In Park (kipark@yuhs.ac) 'Department of Pediatrics, Severance Children's Hospital, Yonsei University College of Medicine, Seoul 03722, Korea

${ }^{2}$ Brain Korea 21 Plus Project for Medical Science, Yonsei University College of Medicine, Seoul 03722, Korea

Full list of author information is available at the end of the article
}

develop translatable neuroprotective and regenerative therapies, the currently available managements for $\mathrm{HI}$ brain injury and SCI are ineffective ${ }^{1-6}$.

Upon implantation into the site of a central nervous system (CNS) injury, multipotent neural progenitor cells (NPCs) not only engraft, migrate toward damaged sites, and differentiate into multiple neural lineages but also provide trophic/immunomodulatory factors and integrate into the remaining host neurons, all of which are promising therapeutic options for neural repair ${ }^{7-14}$. However, NPC-based therapies have shown poor cell survival and integration, as well as either poor differentiation or restricted differentiation into the glial lineages in the host. In addition, to achieve full functional recovery after CNS injury, optimization of cell therapy is needed to recapitulate the precise structural and functional neural wiring 
present in the microenvironment of the $\mathrm{CNS}^{7,13-16}$. Therefore, the efficacy of NPCs for treating CNS injury is currently insufficient, and unexpected side effects have been observed following NPC transplantation ${ }^{7,9,13,14,17}$.

Biomaterials that have already been developed and that are characterized by three-dimensional structure, weblike fibrous morphology, and the distinctive microstructural properties of extracellular matrix (ECM) can enable and facilitate the site-directed delivery of drugs, therapeutic proteins, or stem cells to the CNS, promoting regeneration and repair of damaged neural tissues and circuits $^{9,15,16,18-20}$. Previously, we showed that placement of a fabricated biomaterial scaffold combined with immortalized mouse NPCs (C17.2 cell line) into the infarction cavity of a HI brain injury and a cavity generated by hemisection of the spinal cord reduced parenchymal loss and promoted neurite outgrowth, axonal sprouting, and connectivity ${ }^{9,18}$. Repair of the injured mammalian adult CNS and, in particular, the spinal cord has been a major challenge for neuroscientists. Despite the inhibitory milieu of the adult CNS, the multicomponent, synthetic poly(lactic-co-glycolic acid) (PLGA)-based scaffold of specified architecture seeded with mouse NPCs that acted as a bridge for severe SCI resulted in significant structural and behavioral recovery in adult rats ${ }^{18}$. Additionally, the scaffold alone appeared to reduce inflammation and glial scar formation. Compared with adult SCI, HI brain injury in newborn mouse might offer a permissive environment to reconstitute the injured tissue on its own. However, the postnatal mouse HI brain injury, a well-established model of severe $\mathrm{HI}$ encephalopathy/cerebral palsy in human infants, causes profound tissue damage (a large cystic cavity occupying a significant portion of the cerebral hemisphere ${ }^{11,12}$. Thus, even the most "capable" multipotent NPCs need intrinsic organization, blood supply and a template to guide neural regeneration. Implanted poly(glycolic acid) (PGA) scaffold seeded with mouse NPCs into the infarction cavity facilitated the reciprocal interactions between exogenous graft and HI-injured host brain, and brain tissue reconstitution ${ }^{9}$. However, in that study, the functional recovery after PGA-NPCs complex implantation was not tested.

Despite these positive results, there are concerns over the use of immortalized cells derived from neonatal mouse cerebellum for cell replacement therapies or for neuroprotective support or clinical applications in CNS disorders. Therefore, in this study, we evaluated and compared the regenerative capabilities of biodegradable complexes composed of synthetic PGA scaffold, a clinically safe polymer, seeded with human fetal brain-derived primary NPCs (hNPCs) implanted into the infarction cavity of unilateral HI brain injury in newborn mouse and spinal cord hemisection cavity in adult rat, and assessed motosensory function in the animals after treatment.

\section{Materials and methods}

Human NPC culture and seeding on a fibrous PGA scaffold

Human fetal brain tissue from a cadaver at 13 weeks of gestation was obtained with full parental consent and the approval of the Research Ethics Committee of the Yonsei University College of Medicine, Seoul, Korea (Permit Number: 4-2003-0078) ${ }^{21}$. All procedures conformed to the guidelines of both the National Institutes of Health and the Korean Government. The human NPCs isolated from the telencephalon were grown as neurospheres in serum-free culture medium (DMEM/F12; Gibco, Grand Island, NY) with $\mathrm{N}-2$ supplement (Gibco), $8 \mu \mathrm{g} / \mathrm{ml}$ heparin (Sigma, St. Louis, MO), $20 \mathrm{ng} / \mathrm{ml}$ of fibroblast growth factor 2 (FGF2; R\&D Systems, Minneapolis, MN), and $10 \mathrm{ng} / \mathrm{ml}$ leukemia inhibitory factor (Sigma). hNPCs were passaged every 7-8 days by dissociating the bulk neurospheres with $0.05 \%$ trypsin/EDTA (T/E; Gibco), and cryopreserved at each passage in a Good Manufacturing Practice facility. For scaffold seeding, a 90\% confluent dish of cells was trypsinized and resuspended in 1-2 $\mathrm{ml}$ of $\mathrm{N}-2$ medium $(1 \times \mathrm{DMEM} / \mathrm{F}-12$ and $1 \times \mathrm{N}-2$ supplement; Gibco) at $1.5 \times 10^{7}$ cells $/ \mathrm{ml}$. Then, $100-200 \mu \mathrm{l}$ of the suspension was pipetted onto each piece of PGA scaffold $(\sim 2 \times 2 \times 2 \mathrm{~mm}$ size for HI brain injury; $3 \times 1 \times 1 \mathrm{~mm}$ for SCI; actual bulk density $64.7 \mathrm{mg} / \mathrm{ml}$; Albany Intl. Res. Co., Mansfield, MA) in uncoated dishes, and the constructs were placed in a humidified incubator at $37^{\circ} \mathrm{C}$ with $5 \%$ $\mathrm{CO}_{2}$ for $30 \mathrm{~min}$. Culture medium was then added to the dish and changed every $2-3$ days.

\section{Induction of unilateral HI brain injury in newborn mouse and transplantation of hNPC-PGA complexes into the HI- injured brain}

All procedures were approved by the Institutional Animal Care and Use Committee at Yonsei University College of Medicine. Under isoflurane anesthesia, a unilateral HI brain injury was induced in ICR mice at postnatal day 7 by permanent right common carotid artery occlusion with surgical silk through a ventral midline neck incision. The incision was closed, and the animals were kept warm until they awoke and then returned to their dams for $3 \mathrm{~h}$. The pups were then placed for $1.5 \mathrm{~h}$ in an acrylic chamber with a hypoxic atmosphere $\left(8 \% \mathrm{O}_{2}\right.$ and $92 \% \mathrm{~N}_{2}$ ). Body temperature was maintained at $37^{\circ} \mathrm{C}$ on a warm plate. The sham control group (Sham) underwent anesthesia and incision only.

After having been seeded with hNPCs in culture for 4 days (as described above), two hNPC-PGA complexes were transplanted into the infarction cavity of each mouse brain with microforceps (Fine Science Tools, BC, Canada) 7 days after the induction of HI brain injury. To insert the complex, a triangular portion of the skull overlying the infarction cavity was lifted with a surgical blade, carefully avoiding severing any superficial blood vessels in the brain 
parenchyma. Mice were randomly assigned to four groups: (a) hNPC-PGA implantation $(n=55)$; (b) PGA implantation without hNPCs $(n=55)$; (c) hNPCs $(n=$ $55)$, or $(\mathrm{d})$ vehicle $(\mathrm{N}-2$ medium) injection $(n=55)$. When hNPCs were transplanted, each mouse was, respectively, injected with $12 \mu \mathrm{L}$ of hNPCs $\left(9.6 \times 10^{5}\right.$ cells $)$ into the center of infarcted region of a unilaterally (right-sided) asphyxiated brain using a glass micropipette (diameter, $0.3 \mathrm{~mm}$ ). Pups from three to four different litters were used in each experimental group; male and female mice were equally divided among the experimental groups, and data were obtained in independent experiments. Mice were kept on a $12 \mathrm{~h}$ day/night cycle at $22^{\circ} \mathrm{C}$, fed ad libitum, and maintained in a facility accredited by the Association for Assessment and Accreditation of Laboratory Animal Care. Cyclosporine $(10 \mathrm{mg} / \mathrm{kg}$, i.p.; Sandimmun, Novartis Korea, Seoul) was administered to all groups of mice once per day beginning a day before transplantation until sacrifice.

\section{Hemisection $\mathrm{SCl}$ in adult rat and transplantation of hNPC-PGA complexes into the hemisection cavity}

Adult Sprague-Dawley rats (300-350 g in body weight) were anesthetized with a cocktail of xylazine $(10 \mathrm{mg} / \mathrm{kg}$, i.p.; Bayer Korea, Seoul), ketamine (100 mg/kg i.p.; Yuhan Co., Seoul, Korea) and promazine $(1 \mathrm{mg} / \mathrm{kg}$, i.p.; Samu median, Seoul, Korea). A 4-cm midline incision was done on the back, and the paraspinal muscles were retracted to expose the thoracic laminae. In preliminary experiment, we confirmed that compared with hemisection $\mathrm{SCI}$ at T910 level, SCI at T10-11 level also showed similar deficits in hindlimb locomotor function. Thus, using a dissecting microscope, a laminectomy was performed to expose the dura of the spinal cord at the 10th-11th thoracic $\left(\mathrm{T}_{10}-\mathrm{T}_{11}\right)$ spine vertebrae. The midline of the spinal cord was identified, and one half (the left side) of the spinal cord at the $T_{10}-T_{11}$ level was transected completely over a width of $3 \mathrm{~mm}$ using fine surgical scissors and forceps. After hemostasis was achieved via Gelfoam, a piece of the hNPC-PGA complex previously prepared as described above was transplanted into the hemisection cavity of each rat spinal cord with microforceps. The lesion was confirmed a priori to be similar across all experimental groups and animals. Rats were also randomly assigned to four groups: (a) hNPC-PGA implantation $(n=60)$; (b) PGA implantation without hNPCs $(n=60)$; (c) hNPCs $(n$ $=60)$, or $(\mathrm{d})$ vehicle $(\mathrm{N}-2$ medium) injection $(n=60)$. When hNPCs were transplanted, each rat was respectively injected with $10 \mu \mathrm{L}$ of hNPCs $\left(8.0 \times 10^{5}\right.$ cells $)$ into the hemisection cavity using a glass micropipette (diameter, $0.3 \mathrm{~mm}$ ). After all operations, the musculature was sutured, the skin closed, and the animals were administered cefazolin $(50 \mathrm{mg} / \mathrm{kg}$, i.m.; Chong Kun Dang Pharmaceutical Co., Seoul, Korea) daily for 3 days. During surgery, the rectal temperature was maintained at $37.0 \pm$ $0.5^{\circ} \mathrm{C}$ by a thermostatically regulated heating pad. Ringer's lactate solution $(10 \mathrm{ml})$ was given daily for 3 days post-surgery, and bladders were manually evacuated three times daily until reflex bladder emptying was established. Cyclosporine (10 mg/kg, i.p.; Sandimmun, Novartis Korea, Seoul) was administered daily to all animal groups beginning a day before transplantation until sacrifice. All procedures were approved by the Institutional Animal Care and Use Committee at Yonsei University College of Medicine.

\section{Histopathological analysis}

Histopathology was analyzed after staining with hematoxylin-eosin (H\&E) and Luxol Fast Blue (LFB)-cresyl violet (CV). At 8 weeks after HI brain injury, eight serial coronal sections per mouse (spaced $200 \mu \mathrm{m}$ apart) were stained with H\&E and analyzed with National Institute of Health (NIH) ImageJ Software using images captured via the dotSlide Imaging System and an Olympus BX61VS microscope. The size of each lesion was calculated as the [(area of the left contralateral hemisphere - area of remaining the right ipsilateral hemisphere)/area of the left contralateral hemisphere] $\times 100 \%$. At 8 weeks after SCI, a total four sections per animal (spaced $80 \mu \mathrm{m}$ apart) were stained with $\mathrm{LFB}^{-} \mathrm{CV}$ and calculated with NIH ImageJ software. The atrophy volume was assessed by measuring the area between the reduced actual border of the spinal cord and age-matched intact spinal cord. A demyelination volume was assessed by measuring the area of $\mathrm{LFB}^{-}$/ $\mathrm{CV}^{+}$inside spinal cord. The total lesion volume was defined as the sum of atrophy volume and demyelinated volume $^{22}$.

\section{Immunohistochemistry}

For immunocytochemical analysis, cultured cells were fixed with 4\% paraformaldehyde in PIPES buffer (Sigma), rinsed with phosphate-buffered saline (PBS) solution, blocked with $3 \%$ bovine serum albumin (Sigma), 10\% normal horse serum, and 0.3\% Triton X-100 (Sigma) in PBS. For immunohistochemical analysis, animals were deeply anesthetized, transcardially perfused, and fixed with cold PBS followed by $4 \%$ paraformaldehyde. Brains and spinal cords were removed, post-fixed, cryoprotected in 30\% sucrose, and frozen in O.C.T compound (Sakura Finetek, Torrance, CA). The brains and spinal cords were coronally and/or sagitally sliced into $20-\mu \mathrm{m}$ sections as described ${ }^{23}$. The sections on the slides were stained with following antibodies specific for the following: human nestin (anti-hNestin; 1:200; Chemicon), human cytoplasm (SC121; 1:500; Stem Cells, Inc., Cambridge, UK), human glial fibrillary acidic protein (GFAP) (SC123; 1:500; Stem Cells, Inc., Cambridge, UK), 

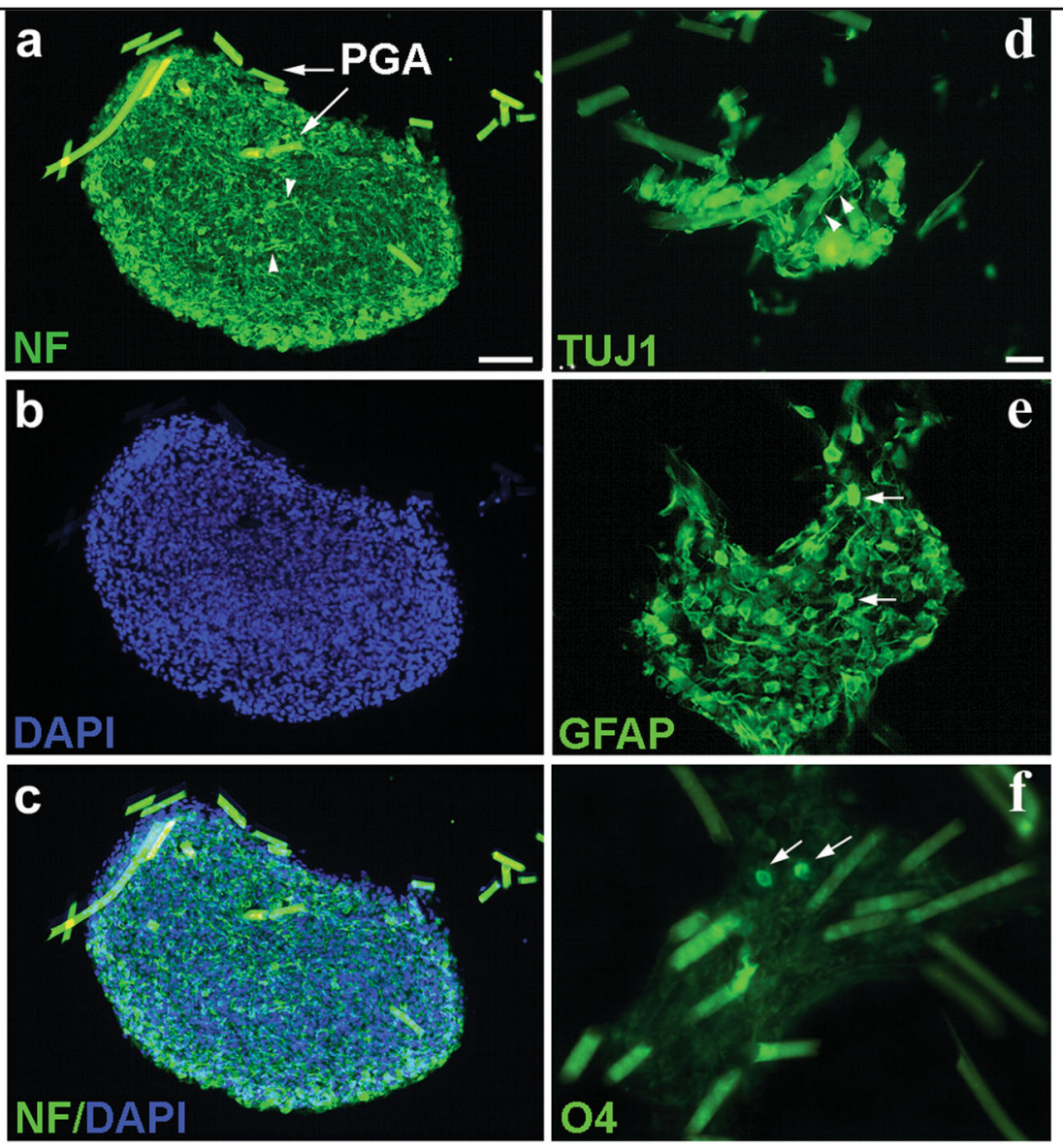

Fig. 1 In vitro characterization of primary hNPCs seeded on a fibrous PGA scaffold. a-d Seven days after being seeded, cells are able to attach, pervade, and grow exuberantly throughout the PGA fibers. The hNPCs predominantly differentiate into $\mathrm{NF}^{+}$and $\mathrm{TUJ}^{+}{ }^{+}$neurons (green) with neuronal processes that adhere to the PGA fibers (arrowheads in $\mathbf{a}, \mathbf{d}$ ). The PGA fibers are indicated by arrows in $\mathbf{a}$. Many of the DAPI ${ }^{+}$hNPCs (b, blue) are co-labeled with anti-NF antibody, seen under dual-filter microscopy in c. e, $\mathbf{f}$ The hNPCs differentiate not only into neurons but also into glial cells. Some human cells express GFAP, an astrocyte/immature cell marker (arrows in e), and a few cells express $\mathrm{O} 4$, an oligodendrocyte progenitor marker (arrows in f). The data shown are representative images. Scale bars: a $100 \mu \mathrm{m} ; \mathbf{d} 10 \mu \mathrm{m}$

glial fibrillary acidic protein (anti-GFAP; 1:1,000; Dako, Glostrup, Denmark), neuronal class III $\beta$-tubulin (antiTUJ1; 1:1,000, Covance, Princeton, NJ), human nuclei (anti-hNuc; 1:100; Chemicon), neurofilament (anti-NF; 1:1,000; Sternberger, Lutherville, MD), O4 (1:50; SigmaAldrich, St. Louis, MO), CNPase (2', $3^{\prime}$-Cyclic-nucleotide 3 '-phosphodiesterase; 1:500: Chemicon), calcitonin gene related peptide (anti-CGRP; 1:1000; Chemicon), choline acetyltransferase (anti-Chat; 1:200; Chemicon), CD31 (1:400; BD Pharmingen), Iba1 (1:500; Wako), CD68 (1:100; Bio-Rad, Hercules, CA), CD86 (1:100; BD Pharmigen), CD206 (1:100; Bio-Rad, Hercules, CA), and von Willebrand Factor (anti-vWF; 1:400; Abcam). Samples were mounted onto slides using Vectashield mounting medium with 4,6-diamino-2-phenylindole (DAPI; Vector), and analyzed by an immunofluorescence microscope (BX51; Olympus, Center Valley, PA) and a Zeiss LSM 700 confocal microscope.

\section{Tract tracing studies}

The tract tracer biotinylated dextran amine conjugated with fluorescein (BDA-FITC) $(0.4 \mu \mathrm{l}$ of $10 \%$ BDA-FITC dissolved in $\mathrm{ddH}_{2} \mathrm{O}$; Vector Laboratories, Burlingame, $\mathrm{CA})$ was stereotactically injected with a glass micropipette at two points in the sensorimotor cortex contralateral to the HI brain injury at or after 10 weeks following administration of the hNPC-PGA complex, PGA alone, hNPCs, or the vehicle ( $n=10$ per group). A glass micropipette was connected via a polyethylene tube to a $10 \mu \mathrm{L}$ Hamilton syringe placed on an infusion pump (KD Scientific, Holliston, MA) and controlled by a microprocessor. All injections were performed over $1 \mathrm{~min}$, and the injection micropipette was kept in place for an additional minute to minimize leakage on withdrawal. Mice were sacrificed 14 days later.

The same volume of BDA-FITC as described above was stereotactically injected with a glass micropipette at four 
points in the sensorimotor cortex contralateral to the side of the spinal cord hemisection at or after 12 weeks following administration of the hNPC-PGA complex, PGA alone, hNPCs, or the vehicle ( $n=10$ per group). Rats were killed 2-4 weeks later. In separate rats, the tract tracer Fast Blue (FB; $1 \mu \mathrm{l}$ of $2 \%$ FB dissolved in $\mathrm{ddH}_{2} \mathrm{O}$ ) (SigmaAldrich) was steretactically injected with a glass micropipette into both the left and right sides of the spinal cord at the $\mathrm{T}_{13}$ level at or after 12 weeks following administration of the hNPC-PGA complex, PGA alone, hNPCs, or the vehicle ( $n=10$ per group). After FB injection, another tract tracer Fast DiI (DiI; $1 \mu$ l of $0.25 \%$ Fast DiI dissolved in dimethylformamide) (Molecular Probes, Eugene, OR) was subsequently injected into both the left and right sides of the spinal cord $3 \mathrm{~mm}$ caudal to the FB injection sites in the same rats. The animals were killed 3 weeks later.

\section{Motosensory functional studies and statistical analysis}

Neurological and accelerating rotarod tests were performed twice a week from 3 to 7 weeks post-transplant in neonatal mice with HI brain injury. Neurological functions were assessed as follows: the ability to stay upright and extend its forelimbs when suspended by its tail, the ability to turn over to rest in a normal position with all 4 feet on the ground after being placed on its side; the ability to place a paw on the surface of a table when it contacts the table edge; and the ability to spread its toes after being suddenly placed or bounced. Each exam was scored as " 0 " if the response was normal and " 1 " if not. The accelerating rotarod test was performed on a beam made of knurled white plastic (diameter: $3 \mathrm{~cm}$, width: 6 cm; LSi Letica; Panlab, Barcelona, Spain), which provides a firm grip. After a mouse was placed on top of the revolving beam $(4 \mathrm{rpm})$ in the orientation opposite to its movement and had become familiarized with the setup, the velocity of the rod was gradually increased to $40 \mathrm{rpm}$ over the 5-min test period. The latency to fall from the rod was calculated from the average of three consecutive trials. All neonatal HI-injured mice were randomized for testing into five experimental groups (Sham, Vehicle, PGA, hNPCs, and hNPCs-PGA groups; $n=15$ per group) by a researcher blinded to the treatment group.

Hindlimb locomotor performance was assessed by the open-field Basso, Beattie, and Bresnahan (BBB) scoring system $^{24}$ in rats with SCI. A rat was placed for $5 \mathrm{~min}$ at the center of a circular open field (diameter: $90 \mathrm{~cm}$, wall height: $7 \mathrm{~cm}$ ) with a non-slip floor. The locomotor activity of the ipsilateral lesioned, and contralateral unlesioned, hindlimbs was monitored 1 week after the injury and weekly for 8 weeks after transplantation. Each score represents a distinct motor functional state from 0 (complete paralysis) to 21 (normal mobility) through joint movements, stepping ability, coordination, and trunk stability. The examination was performed by three observers who were not aware of the treatment received by each rat. All SCI rats were block-randomized into four experimental groups (Vehicle, PGA, hNPCs, and hNPCs-PGA group; $n=15$ per group) based on BBB scores recorded on week 1 to ensure equivalent deficits across the experimental groups before starting the transplantation.

Hindpaw mechanical allodynia was assessed by measuring withdrawal response to mechanical stimulation with von Frey filaments (Stoelting, Wood Dale, IL) placed on the mid plantar surface of the ipsilateral hindpaw. All rats were tested for mechanical threshold prior to surgery and on 14, 28, 42, and 56 days after transplantation. Before testing, rats were housed in the test cage above a metal mesh and acclimatized for $30 \mathrm{~min}$. The $50 \%$ withdrawal mechanical threshold was measured by using Dixon's up and down method ${ }^{25}$. A series of von Frey filaments (log unit; 3.61, 3.84, 4.08, 4.31, 4.56, 4.74, 4.93 and 5.18) were applied serially for 10-s inter stimulus intervals to the hindpaw in six applications, beginning with the $4.31 \log$ unit von Frey filament.

For statistical analysis, IBM SPSS Software, version 20, was used. Behavioral data were assessed using a repeated measures analysis of variance. Whenever a treatment was found to have statistically different result over time or a treatment effect was observed, with a $p$-value of less than 0.05 , the Bonferroni post hoc procedure was used for pairwise comparison of groups. Sample sizes were calculated using power analyses with an $\alpha$-level of 0.05 and a power of 0.8 . The data represent the means \pm standard error of the mean (SEM). Differences were considered statistically significant at $p$-values of $<0.05$.

\section{Results}

\section{Characterization of hNPC-PGA complex in vitro}

In this study, a scaffold of PGA fibers $(10-15 \mu \mathrm{m}$ in diameter) assembled into a woven array was used ${ }^{26-28}$. To investigate the attachment, growth, migration, and differentiation of progenitor cells on the polymer scaffold, a suspension of primary hNPCs was seeded onto PGA fibers in uncoated plastic dishes. After 7 days, the cells attached, pervaded, migrated, and grew exuberantly throughout the porous PGA fibers (Fig. 1a-c), indicating the absence of PGA neurotoxicity ( $\sim 99 \%$ survived; $n=5$ ). The cells not only grew and flourished but also differentiated into neurons and glial cells (Fig. 1d-f). More than $70 \%$ of the cells showed the increased expression of the neuronal marker NF (Fig. 1a, green) with neuronal processes (arrowheads in Fig. 1a) and the early neuronal cell marker TUJ1 with neurite extension (arrowheads in Fig. $1 \mathrm{~d} ; n=5$ per group), while $\sim 30 \%$ expressed GFAP, an astrocyte/immature progenitor cell marker (arrows in Figs. 1e), and $\sim 1-2 \%$ expressed O4, an oligodendrocyte 
marker (arrows in Fig. 1f; $n=5$ per group). Cells undergoing neuronal differentiation extended long neurites that adhered to, stretched along, enwrapped, and interconnected with the PGA fibers (arrowheads in Fig. 1d). These findings demonstrate that PGA fibers can support hNPC survival, attachment, neuronal and glial differentiation, and neurite outgrowth, suggesting their suitability as an implantable substrate for hNPCs in vivo within injured neural tissue.

\section{Transplantation of hNPC-PGA complexes into the infarction cavity of a neonatal $\mathrm{HI}$ brain injury and neuroregenerative response}

Permanent ligation of the right common carotid artery followed by exposure to $8 \% \mathrm{O}_{2}$ in 1-week-old mice leads to extensive infarction of the ipsilateral cortex, hippocampus, striatum, and/or thalamus, leading to the evolution of large cystic cavities in the cerebral hemisphere (asterisks [*] in Fig. 2a) while leaving the contralateral hemisphere grossly intact. It is thus a well-established model of severe focal neonatal HI brain injury ${ }^{29}$. To investigate in vivo tolerance and the regenerative response, hNPC-PGA complexes, maintained in culture for 5 days, were implanted into the evolving infarction cavity 7 days post-HI brain injury $(n=55)$. The hNPC-PGA complex refilled the cavity, significantly reduced parenchymal loss (Fig. 2a and Fig. 4a-d), and appeared to integrate into the injured cerebrum. By 2 weeks post-transplantation $(n=15)$, the PGA fibers began to biodegrade (green strands, arrowheads in Fig. 2a), and the cavity seemed to be filled with de novo tissue. Immunohistochemical analysis of the de novo tissue within the infarction cavity revealed that donorderived cells, recognized by their robust hNestin expression (Fig. 2a, green) were well engrafted within the cavity, and some of them differentiated into neurons $(\sim 20 \%$; Fig. 2b-d), which had extended neuronal processes (arrows in Fig. 2c, d) that ran along the residual PGA fibers (arrowheads in Fig. 2b-d) and GFAP $^{+}$cells $(\sim 35 \%$; Fig. $2 \mathrm{e}-\mathrm{g}$ ), although most cells expressed nestin $(>80 \%)$ immature cell marker at this time. The sum of all quantification markers was more than $100 \%$, suggesting that there is an overlap between some cell markers. Nestin, in particular, has been found to colocalize with $\beta$-tubulin III, GFAP, and the oligodendroglial progenitor marker Olig2 $2^{30}$. Additionally, GFAP expression did not always mean the acquisition of an astroglial fate in hNPCs because GFAP-positive cells also represent immature $\mathrm{hNPCs}^{31}$. These findings mean that some of the grafted cells in this study express both neuronal (NF) and glial cell/immature cell (GFAP) marker suggesting their immature state in vivo. Furthermore, donor-derived cells have rarely been observed more than 2 months after transplantation. Implanted hNPCs appear not to survive well for a long time in highly inflammatory, inhospitable HI-injured brain following xenotransplantation despite the use of immunosuppressant. Hence, although implanted hNPCs seeded on PGA scaffold appear capable of repopulating damaged brain regions, the vast volume of tissue loss following the severe cases of brain injury would seem to preclude differentiation of grafted cells and even partial replacement.

In histopathological analysis at 8 weeks posttransplantation (Supplementary Figure S1a, b), H\&Estained bran sections showed complete degradation of the PGA scaffold after HI brain injury. Based on assessment of tissue sparing in brains of each group $(n=10$ per group), the infarction volume was significantly decreased in hNPC-PGA complex-transplanted vs. vehicle-injected mice $(34.3 \% \pm 6.0 \%$ vs. $60.9 \% \pm 3.8 \%, P<0.05)$, whereas PGA alone $(47.8 \% \pm 4.3 \%)$-treated or hNPC $(50.2 \% \pm$ 6.3\%)-treated mice exhibited no difference from the vehicle group. Hence, hNPC-PGA complex transplantation effectively reduces infarct volume in $\mathrm{HI}$ brain injury. By 8 weeks post-transplantation $(n=15)$, the newly formed tissue had become highly vascularized (arrowheads in Fig. 4d and e) and appeared to be connected to the host tissue by an intricate network of multiple branching neuronal fibers and processes from both hostderived and donor-derived neurons (arrowheads in Fig. $2 \mathrm{~h}-\mathrm{k}$ ). However, this event was not obvious in the control groups treated with PGA alone, hNPCs, or the vehicle after $\mathrm{HI}$ brain injury, as noted later (Fig. $4 \mathrm{~h}$ and $\mathrm{k}$; $n=15$ per group). These findings suggest that donorderived tissue not only integrate into host tissue but also intimately communicate with each other, including through activation of angiogenic-, neurite extensionpromoting, and axonal outgrowth-promoting signals.

To determine whether the hNPC-PGA complex implanted into the infarction cavity established such longdistance neuronal connections with some of their probable target regions, tract tracer BDA-FITC was injected into the contralateral intact cortex (within the presumptive target region of the transcallosal sensorimotor fibers) of one group of animals $(n=10)$ at or after 10 weeks post-transplantation. Analysis 14 days later showed that fluorescein-labeled axons in the cerebral cortex and external capsule of the intact hemisphere, projecting through the corpus callosum and toward the implantation site of the hNPC-PGA complex within the injured ipsilateral cortex and penumbra (Fig. 2l). Some processes labeled with BDA-FITC in an anterograde manner from neurons in the contralateral intact hemisphere were observed sprouting their axons toward the site where the hNPC-PGA complex was implanted in the new "integrated" cortical parenchyma. In addition, the cell body and cellular processes of a neuron-like cell in the ipsilateral cortical penumbra, labeled with BDA-FITC in a 

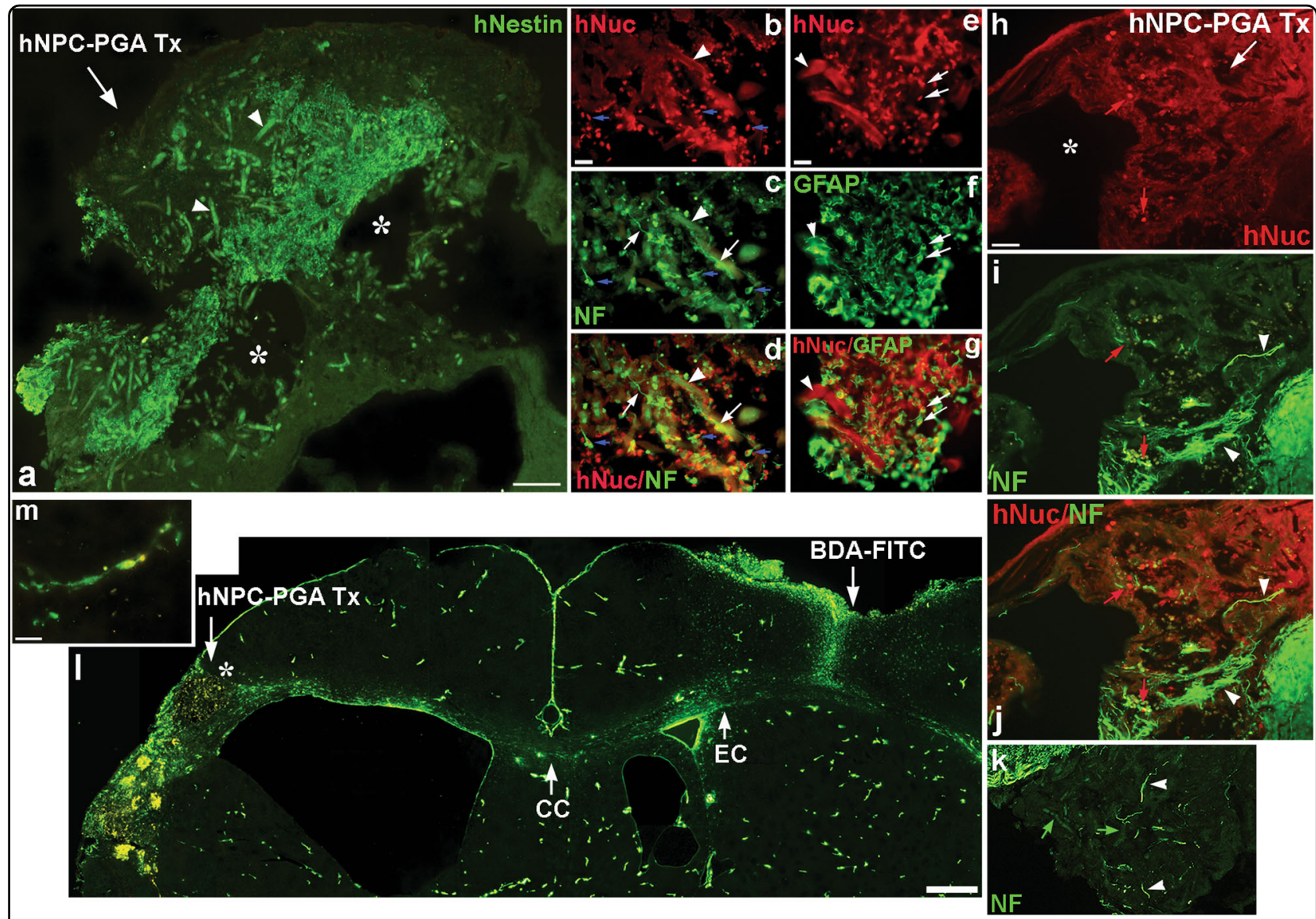

Fig. 2 Transplantation of hNPC-PGA complexes into the infarction cavity of a HI brain injury. a Two weeks after transplantation (Tx) of the hNPC-PGA complexes (an arrow) into the infarction cavity indicated by asterisk, hNestin ${ }^{+}$donor-derived cells (green) show robust engraftment within the injured area. The PGA fibers have begun to biodegrade (green strands, arrowheads). $\mathbf{b}-\mathbf{g}$ Some hNuc donor-derived cells (red in $\mathbf{b}$, $\mathbf{d}$, e, g) show co-localization with NF (blue arrows in $\mathbf{b}, \mathbf{c}, \mathbf{d}$ ) with extended neuronal processes (white arrows in $\mathbf{c}$, $\mathbf{d} ; n=15$ ) or GFAP (white arrows in $\mathbf{e}, \mathbf{f}$ $\mathbf{g} ; n=15)$. The PGA fibers are indicated by arrowheads in $\mathbf{b}-\mathbf{g}$. $\mathbf{h}-\mathbf{k}$ The de novo tissue following Tx of the hNPC-PGA complex into the infarction cavity (an asterisk in $\mathbf{h}$ ) is connected to the host brain tissue by multiple $\mathrm{NF}^{+}$neuronal processes (arrowheads in $\mathbf{i}, \mathbf{j}$, $\mathbf{k}$ ) from host-derived and/or donor-derived neurons. Some donor-derived cells are identified by anti-hNuc staining (red arrows in $\mathbf{h}, \mathbf{l}, \mathbf{j}$ ). The PGA fibers are indicated by green arrows in $\mathbf{k}$. I, $\mathbf{m}$ The tract tracer BDA-FITC was injected into the contralateral intact cortex at 10 weeks following implantation of the hNPC-PGA complex into the infarction cavity. Axonal projections labeled green with fluorescein can be visualized from the cerebral cortex and external capsule (EC) of the intact hemisphere, projecting through the corpus callosum (CC) and toward the implantation site of the hNPC-PGA complex within the injured ipsilateral cortex and penumbra (I). Some BDA-FITC + anterograde-labeled processes from neurons in the contralateral intact hemisphere are observed sprouting their axons toward the implantation site of the hNPC-PGA complex (I). The BDA-FITC ${ }^{+}$retrograde-labeled cell body and cellular processes of a neuron-like cell in the ipsilateral cortical penumbra indicated by an asterisk (in $\mathbf{I}$ ) are well visualized at higher magnification ( $\mathbf{m}$ ). The data shown are representative images. Scale bars: a, I $500 \mu \mathrm{m} ; \mathbf{b}, \mathbf{e}, \mathbf{h}, \mathbf{m} 20 \mu \mathrm{m}$

retrograde manner and indicated by an asterisk $(*)$, were well visualized at higher magnification (Fig. $2 \mathrm{~m}$ ). However, long-distance neuronal extension could not be seen when PGA fibers alone, hNPCs, or vehicle were administered into HI-injured brain regions. These data suggest that the combination of hNPCs and the polymer scaffold appears to play an important role in the reformation of long-distance connections between donor-derived or host neurons and their targets in both cerebral hemispheres in some instances.
Transplantation of hNPC-PGA complexes into the hemisection cavity of an adult $\mathrm{SCl}$ and the resulting neuroregenerative response

A piece of hNPC-PGA complex, maintained in culture for 5 days as described above, was implanted into the hemisection cavity of the spinal cord immediately after the induction of SCI $(n=60)$. As with the HI brain injury, the hNPC-PGA complex not only appeared to refill the cavity and compensate for parenchymal loss but also became integrated into the injured spinal cord at 4 weeks post-transplantation (Fig. $3 \mathrm{a}$ and b; $n=15$ ). In 


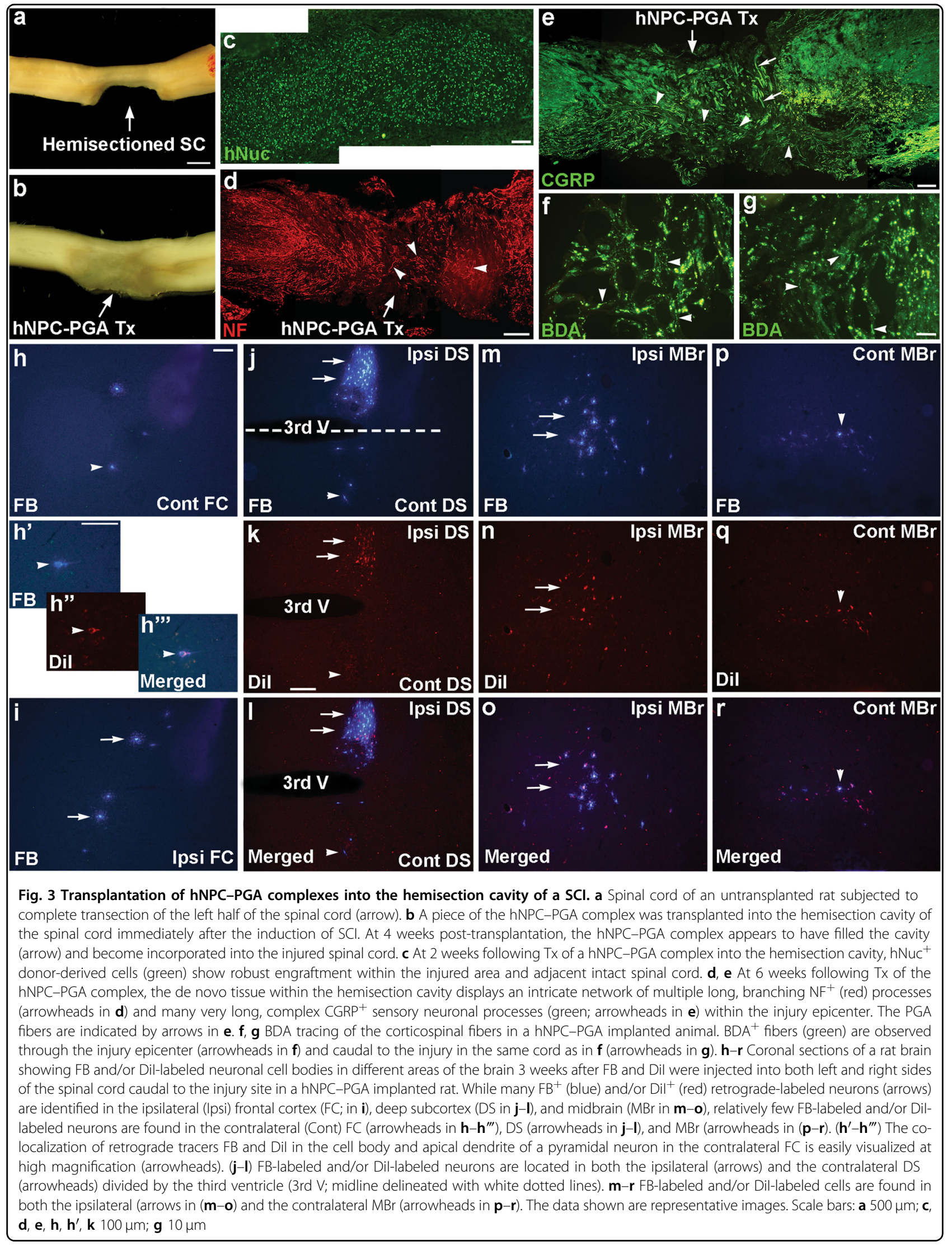



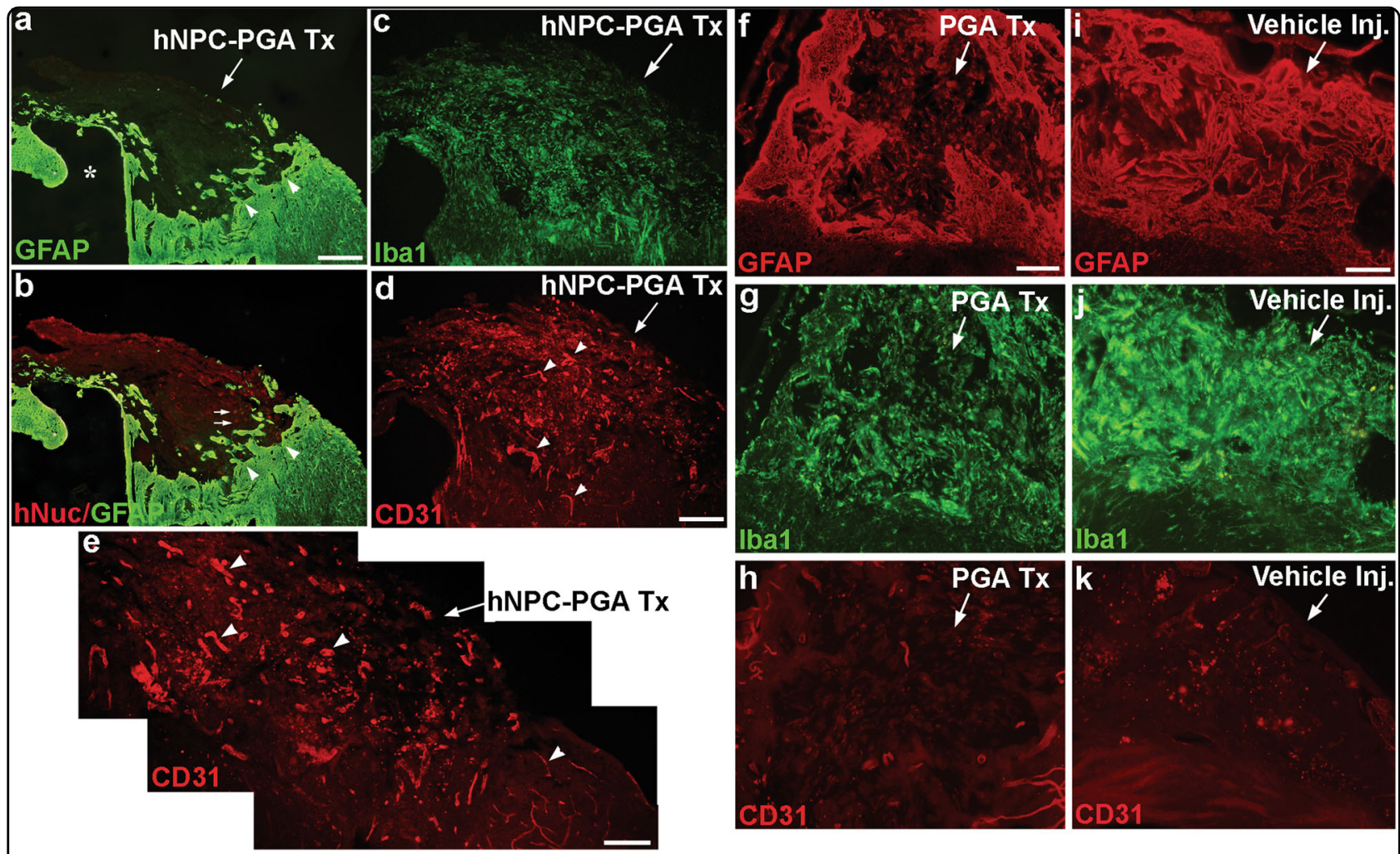

Fig. 4 Implantation of a hNPC-PGA complex into HI brain injury inhibits glial scar formation and the gliotic response and increases neovascularization. $\mathbf{a}$, $\mathbf{b}$ The hNPC-PGA Tx into the infarction cavity, indicated by an asterisk (in $\mathbf{a}$ ), leads to a significant decrease in the density of GFAP staining (green) at the lesion epicenter, while there is intense GFAP staining in the peri-infarct area at 8 weeks post-transplantation (arrowheads in $\mathbf{a}, \mathbf{b}$ ). A few of hNuc ${ }^{+}$donor-derived cells (red) are found within the newly formed tissue by implantation of the hNPC-PGA complex (arrows in $\mathbf{b}$ ). $\mathbf{f}, \mathbf{i}$ The PGA-alone Tx also causes a significant decrease in GFAP staining (red) in the injury epicenter (an arrow in $\mathbf{f}$ ), while there is highly increased GFAP staining (red) in the injury epicenter and peri-infarct area of vehicle-injected (Vehicle Inj.) animal at 8 weeks post-injection (an arrow in i). $\mathbf{c}, \mathbf{g}, \mathbf{j}$ Tx of hNPC-PGA or PGA-alone into the infarction cavity causes less Iba1 staining (green) in the injury epicenter than vehicle injection (arrows in $\mathbf{c}, \mathbf{g}, \mathbf{j}$, respectively). $\mathbf{d}, \mathbf{e}, \mathbf{h}, \mathbf{k}$ At the same time post-transplantation, hNPC-PGA Tx causes highly significantly more CD31 ${ }^{+}$(red) small blood vessels in the injury epicenter (arrowheads in $\mathbf{d}$, e) than implantation of PGA alone and injection of the vehicle (arrows in $\mathbf{h}, \mathbf{k}$ ). The data shown are representative images. Scale bars: a, d $500 \mu \mathrm{m} ; \mathbf{e}, \mathbf{f}, \mathbf{i} 200 \mu \mathrm{m}$

histopathological analysis at 8 weeks post-transplantation (Supplementary Figure S1c, d), LFB-CV-stained spinal cord sections showed complete degradation of the PGA scaffold after SCI. Based on assessment of tissue sparing in spinal cords of each group ( $n=10$ per group), the total lesion volume (atrophy volume + demyelination volume) was significantly decreased in hNPC-PGA-transplanted vs. vehicle-injected rats $\left(3.1 \mathrm{~mm}^{3} \pm 0.4 \mathrm{~mm}^{3}\right.$ vs. $5.2 \mathrm{~mm}^{3}$ $\left.\pm 0.6 \mathrm{~mm}^{3}, P<0.05\right)$, whereas PGA alone $\left(4.0 \mathrm{~mm}^{3} \pm 0.2\right.$ $\left.\mathrm{mm}^{3}\right)$-transplanted or hNPC $\left(4.4 \mathrm{~mm}^{3} \pm 0.2 \mathrm{~mm}^{3}\right)$-transplanted rats demonstrated no difference from vehicle group. We also quantified demyelination volume. However, hNPC-PGA-, PGA alone-, hNPCs-, and vehicletreated rats showed no difference in SCI-induced demyelination volume $\left(1.2 \mathrm{~mm}^{3} \pm 0.5 \mathrm{~mm}^{3} ; 1.5 \mathrm{~mm}^{3} \pm\right.$ $0.1 \mathrm{~mm}^{3} ; 1.7 \mathrm{~mm}^{3} \pm 0.2 \mathrm{~mm}^{3}$; and $1.6 \mathrm{~mm}^{3} \pm 0.7 \mathrm{~mm}^{3}$, respectively). Hence, hNPC-PGA complex transplantation shows the significant neuroprotection against $\mathrm{SCI}$, but do not effectively reduce demyelination volume.
Immunohistochemistry demonstrated that donor-derived cells, recognized by their robust hNuc expression (Fig. 3c), were well engrafted within the hemisection cavity. They migrated toward adjacent intact spinal cord segments, and a few of them differentiated into TUJ1 $1^{+}$neurons ( $\left.9 \%\right)$, $\mathrm{GFAP}^{+}$astrocytes $(\sim 4 \%)$ and CNPase ${ }^{+}$oligodendrocytes ( $\sim 2 \%)$, though most donor cells remained in an immature hNestin-positive state ( $>80 \%$ ) (Supplementary Figure S2). Hence, although implanted hNPCs seeded on PGA scaffold appear capable of repopulating lesion sites, the sheer volume of tissue loss and inhibitory milieu of the adult injured spinal cord would seem to preclude differentiation of the grafted cells and even partial replacement. By 6 weeks post-transplantation $(n=10)$, the de novo tissue consisting of the hNPC-PGA complex within the hemisection cavity exhibited many long, complex $\mathrm{NF}^{+}$neurite outgrowths (Fig. 3d) and became highly vascularized (Fig. 5d), suggesting that the newly formed tissue was connected by neuronal fibers and processes with host 

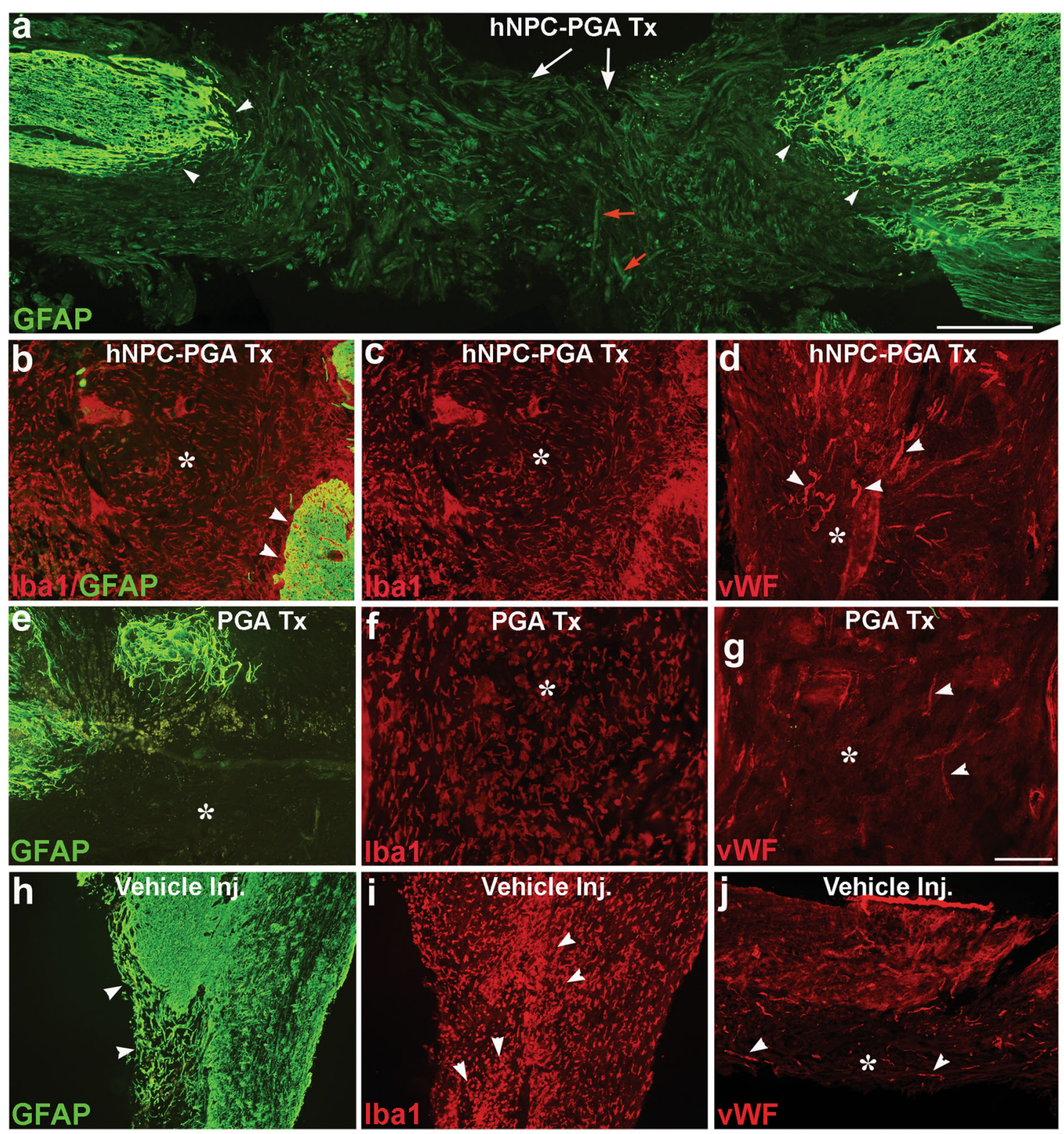

Fig. 5 Implantation of a hNPC-PGA complex into a SCI inhibits glial scar formation and the gliotic response and increases neovascularization. $\mathbf{a}$, $\mathbf{b}$ Tx of hNPC-PGA into the hemisection cavity causes a significant decrease in the density of GFAP staining (green) at the lesion epicenter (white arrows in $\mathbf{a}$; asterisk in $\mathbf{b}$ ), while there is intense GFAP staining in the perilesion area at 6 weeks post-transplant (arrowheads in $\mathbf{a}, \mathbf{b}$ ). The PGA fibers are indicated by red arrows in $\mathbf{a}$. $\mathbf{e}, \mathbf{h}$ Tx of PGA alone also leads to significantly lower GFAP staining (green) in the injury epicenter (asterisk in $\mathbf{e}$ ), while there is significantly more GFAP staining (green) in the injury epicenter and perilesion area of the vehicle-injected (Vehicle Inj.) animal at 6 weeks post-injection (arrowheads in $\mathbf{h}$ ). $\mathbf{c}, \mathbf{f}, \mathbf{i}$ Tx of hNPC-PGA and PGA alone into the hemisection cavity causes less Iba1 staining (red) in the injury epicenter (asterisk in $\mathbf{c}, \mathbf{f}$ ), while there is highly increased Iba1 staining (red) in the injury epicenter and perilesion area of the vehicle-injected animal at 6 weeks post-injection (arrowheads in $\mathbf{i})$. (d, $\mathbf{g}, \mathbf{j})$ At the same time post-transplantation, hNPC-PGA Tx causes highly significantly more $\mathrm{WWF}^{+}$(red) small blood vessels (arrowheads in $\mathbf{d}$ ) in the injury epicenter (asterisk in $\mathbf{d}$ ) than implantation of PGA alone or injection of the vehicle (arrowheads in $\mathbf{g}, \mathbf{j}$ ). An asterisk (* in $\mathbf{g}, \mathbf{j}$ ) indicates the injury epicenter. The data shown are representative images. Scale bars: a $500 \mu \mathrm{m}$; g $200 \mu \mathrm{m}$

spinal cord and well vascularized. However, donorderived cells identified in the spinal cord both rostral and caudal to the injury epicenter were not immunopositive for NF (Supplementary Figure S3a-c) but immunopositive for hNestin (Supplementary Figure S2), suggesting that the extensive neurite outgrowth in the new parenchyma was actually of host origin. In fact, an intricate network of multiple branching $\mathrm{NF}^{+}$processes were present within the hNPC-PGA complex and its parenchyma running along the length of the dissolving 
PGA fibers (Supplementary Figure S3d-f). In addition, to examine whether implantation of the hNPC-PGA complex promotes sensory and motor axon regeneration, the growth of sensory and motor neuronal processes within the injury epicenter were assessed using immunostaining with anti-CGRP and Chat antibodies, respectively. Many exceedingly long, complex $\mathrm{CGRP}^{+}$processes (Fig. 3e) and some $\mathrm{Chat}^{+}$neuronal processes (Supplementary Figure S4) were observed within the new parenchyma, showing evidence of the regeneration of sensory and motor spinal axons through the hNPC-PGA complex "bridges". By contrast, the control groups treated with PGA alone or the vehicle post-SCI ( $n=10$ per group) showed much less neovascularization (Fig. $5 \mathrm{~g}$ and $\mathrm{j}$ ) and neurite extension throughout the injured region of the cord. These findings suggest that implantation of the hNPC-PGA complex remarkably improves integration and communication between donor-derived tissue and host tissue in SCI as well as $\mathrm{HI}$ brain injury.

To examine whether the hNPC-PGA complex implanted into a SCI establishes a corticospinal tract (CST), BDA-FITC was injected into the sensorimotor cortex contralateral to the side of the spinal cord hemisection of a group of animals $(n=10)$ at or beyond 12 weeks post-transplantation. Analysis 2-4 weeks later demonstrated that fluorescein-labeled CST axons were detected through the injury epicenter (Fig. 3f and Supplementary Figure S5) and caudal to the injury (Fig. 3g and Supplementary Figure S5). BDA, better known as an anterograde neuronal tracer, had been transported along either spared or regenerated corticospinal fibers. By contrast, administration of PGA fibers alone, hNPCs, or the vehicle ( $n=10$ per group) showed positive BDA tracing rostral but not caudal to the injury epicenter (Supplementary Figure S6). As an independent verification of these findings, we used retrograde neuronal tracers, FB and Dil, to label cell bodies of neurons with axons projecting into or through the injury epicenter of the spinal cord following implantation of the hNPC-PGA complex. $\mathrm{FB}$ and then DiI were injected into the left and right sides of the spinal cord, respectively, caudal to the injury site in the same rats $(n=10)$ at or after 12 weeks posttransplantation. Three weeks later, FB-labeled and/or DiI-labeled neuron cell bodies through both the right intact and left injured cords were observed in multiple areas of the brain (Fig. 3h-r). Many neurons labeled with FB and/or DiI in a retrograde manner were identified in the ipsilateral frontal cortex, deep subcortical region, and midbrain to the spinal cord hemisection (Fig. 3i, j-l, $\mathrm{m}-\mathrm{o}$ ). By contrast, relatively few neurons labeled with FB and/or DiI were found in the contralateral frontal cortex, deep subcortex, and midbrain to SCI (Fig. 3h-h'", j-l, $\mathrm{p}-\mathrm{r})$. In control animals treated with PGA alone, hNPCs, or the vehicle ( $n=10$ per group), no labeled neurons and axons were observed in the side of the brain contralateral to the spinal cord hemisection (Supplementary Figure S6). In tract tracing studies, it may be possible that axons crossing hNPC-PGA graft placed at the lesion site, and rostral or caudal graft-host interface could have arisen from spared ventral or lateral corticospinal axons. However, to prove the CST regeneration, further research is required to confirm the electrophysiological connectivity generated by the regenerating corticospinal axons within the graft and beyond spinal cord lesion sites. Hence, these findings suggest that hNPC-PGA graft may protect some injured corticospinal fibers.

\section{Implantation of the hNPC-PGA complex inhibits glial scar formation, alters the gliotic response, and increases neovascularization}

Following CNS injury, the formation of a glial scar creates a physical and molecular barrier to regeneration. Reactive astrocytes are the major component of the glial scar, which inhibits axonal regeneration and functional recovery ${ }^{32}$. In this study, we used immunostaining to analyze GFAP expression surrounding the HI brain injury site at 8 weeks $(n=15)$ and SCI site at 6 weeks $(n=10)$ post-transplantation. The group with hNPC-PGA implanted in HI brain injury showed significantly lower density of GFAP staining at their lesion epicenters (Fig. 4a, b) than the vehicle-injected control group (Fig. 4i). The newly formed tissue within the infarction cavity showed almost no GFAP staining, while there was intense immunostaining in the peri-infarct area around the perimeter of the implants (Fig. 4a, b). The PGA-alone group exhibited significantly less GFAP staining in the injury epicenter than the vehicle-injected group (Fig. 4f, i; $n=15$ per group).

In the SCI experiment, the hNPC-PGA group as well as PGA-alone group showed highly significantly lower density of GFAP staining at their lesion epicenters (Fig. 5a, b, e) than the vehicle-injected group (Fig. $5 \mathrm{~h} ; n=10$ per group). The newly formed tissue within the hemisection cavity showed almost no GFAP staining, while there was intense immunostaining in the peri-injury area around the perimeter of the implants (Fig. 5a, b, e). These results suggest that hNPC-PGA complex or PGA alone implantation significantly reduces GFAP-positive astrocyte infiltration after CNS injury, in turn reducing astrogliosis and glial scar formation.

To examine whether hNPC-PGA complex implantation would alter microglia/macrophage response to CNS injury, immunostaining for Iba1, CD68 (activated microglia/macrophage marker), CD86 (marker for M1 polarization of microglia/macrophage), and CD206 (marker for M2 polarization of microglia/macrophage $)^{33,34}$ was performed at 8 and 6 weeks post-transplantation into the HIinjured brain and injured cord, respectively. In both brain 

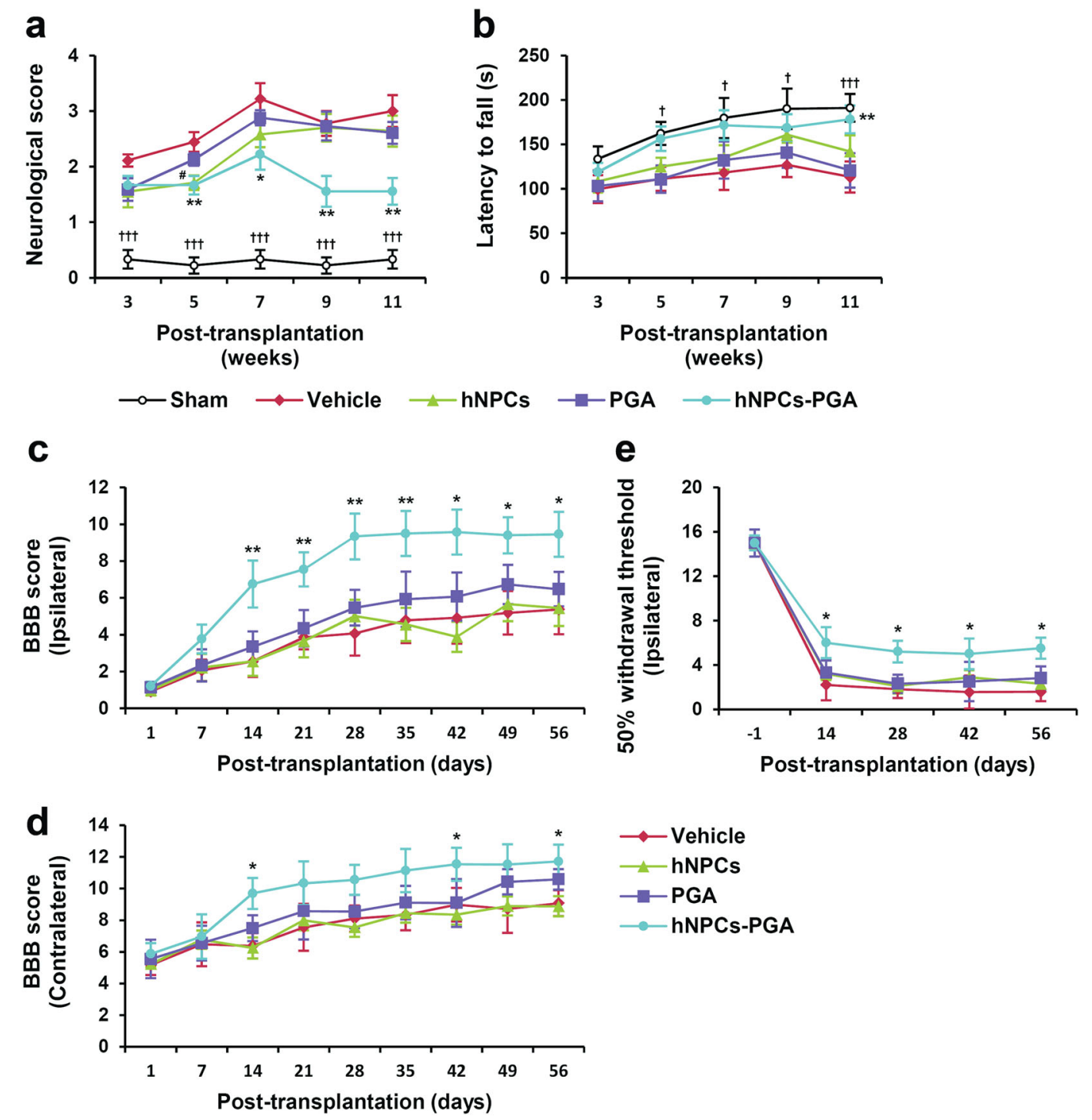

Fig. 6 Behavioral performance of animals treated with hNPC-PGA, PGA alone, hNPCs, or vehicle after HI brain injury or SCl. a, b The results of the neurological test (a) and the latency to fall in the accelerating rotarod task (b) in HI brain injury are shown at 3, 5, 7, 9, and 11 weeks posttransplantation. $\mathbf{a}^{*}$ and ${ }^{* *}$ indicate a significant difference between the hNPC-PGA-treated and vehicle-treated groups, ${ }^{*}$ exhibits a significant difference between hNPC-treated and vehicle-treated groups, and ${ }^{+++}$represents a significant difference between sham controls and vehicleinjected group. $\mathbf{b}^{* *}$ indicates a significant difference between the hNPC-PGA-treated and vehicle-treated groups, and ${ }^{+}$and ${ }^{+++}$represent a significant difference between sham controls and vehicle-injected group. $\mathbf{c}$, $\mathbf{d}$ The BBB open-field walking scores for the ipsilateral lesioned (c) and contralateral unlesioned hindlimbs (d) after SCl are shown at 1, 7, 14,21, 28, 35, 42, 49, and 56 days post-transplantation. * and ** indicate a significant difference between the hNPC-PGA-treated and vehicle-treated groups in $\mathbf{c}$, $\mathbf{d}$. e Von Frey test for mechanical allodynia on ipsilateral lesioned hindlimbs after $\mathrm{SCl}$ are shown at a day before $\mathrm{SCl}$, and $14,28,42$, and 56 days post-transplantation. The data represent the mean \pm SEM. $\left({ }^{*},{ }^{+} P<0.05\right.$ vs. vehicle; ${ }^{* *} P<0.01$ vs. vehicle; $\left.{ }^{+++} P<0.001\right)$

injury and the SCI, the hNPC-PGA-implanted and PGA alone-implanted groups exhibited less Iba1 and CD68, and more CD206 staining in the injury epicenter than the vehicle-injected group (Fig. 4c, g, j; Fig. 5b, c, f, i; and Supplementary Figure S7 and S8). Additionally, the hNPC-PGA graft group showed less CD86 staining than that of vehicle control in $\mathrm{HI}$ brain injury (Supplementary
Figure S7d, f). Thus, these data indicate that hNPC-PGA complex or PGA alone implantation may reduce microglia/macrophage infiltration following CNS injury and change polarization of microglia/macrophage from M1 to M2 type, which is considered to be beneficial for neural repair $^{33,34}$. 
As noted earlier, to investigate the effect of implanting the hNPC-PGA complex into brain injury or SCI on neovascularization of the de novo tissue within the lesion cavity, immunostaining for CD31 and vWF was performed. At 8 weeks post-transplantation, the hNPC-PGA group showed highly significantly more $\mathrm{CD}^{+} 1^{+}$blood vessels in the $\mathrm{HI}$-injured brain than the groups treated with PGA-alone or the vehicle (Fig. 4d, e, h, and k). At 6 weeks post-transplantation into a SCI, the hNPC-PGA group also demonstrated many more $\mathrm{vWF}^{+}$blood vessels than the PGA-alone and vehicle-only groups (Fig. 5d, g, j). These findings suggest that implantation of hNPC-PGA complex provides structural support for hNPCs, as well as promote a neovascularization in the HI brain and SCI.

\section{Implantation of the hNPC-PGA complex facilitates motosensory recovery}

We evaluated the therapeutic potential of the implanted hNPC-PGA to improve neurological and motor functions in neonatal mice with $\mathrm{HI}$ brain injury. Vehicle-injected mice showed significant higher impairment of neurological function than the sham controls at 3, 5, 7, 9, and 11 weeks post-injection $(2.11 \pm 0.11$ vs. $0.33 \pm 0.17, P<$ $0.001 ; 2.44 \pm 0.18$ vs. $0.22 \pm 0.15, P<0.001 ; 3.22 \pm 0.28$ vs. $0.33 \pm 0.17, P<0.001 ; 2.78 \pm 0.22$ vs. $0.22 \pm 0.15, P<0.001$; and $3.00 \pm 0.29$ vs. $0.33 \pm 0.17, P<0.001$, respectively; $n=$ 15 per group; Fig. $6 a)$. Mice treated with hNPC-PGA ( $n$ $=15$ ) demonstrated significant improvement in the sensory-motor response in neurological tests at 5, 7, 9, and 11 weeks post-transplantation compared with the vehicle-injected mice $(1.67 \pm 0.17$ vs. vehicle, $P<0.01$; $2.22 \pm 0.28$ vs. vehicle, $P<0.05 ; 1.56 \pm 0.28$ vs. vehicle, $P<$ 0.01 ; and $1.56 \pm 0.24$ vs. vehicle, $P<0.01$, respectively; Fig. 6a). However, hNPC-grafted mice showed significant improvement only at 5 weeks after transplantation (1.71 \pm $0.13, P<0.05$ vs. vehicle injection) and PGA aloneimplanted mice ( $n=15$ per group) showed no differences from the vehicle-injected mice (Fig. 6a). In the accelerating rotarod task, vehicle-injected mice at 5, 7, 9 and 11 weeks post-injection showed significantly lower motor coordination than the sham controls (111.33 \pm 13.54 vs. $162.37 \pm 13.03 \mathrm{~s}, \quad P<0.05 ; \quad 118.30 \pm 19.41$ vs. $179.67 \pm 22.54 \mathrm{~s}, \quad P<0.05 ; \quad 126.89 \pm 13.71$ vs. $190.07 \pm$ 22.83 s, $P<0.05$; and $113.37 \pm 17.41$ vs. $191.04 \pm 15.56$ s, $P$ $<0.001 ; n=15$ per group; Fig. $6 \mathrm{~b})$. The mice treated with hNPC-PGA $(n=15)$ showed a significantly higher latency to fall at 11 weeks post-transplantation than vehicle-injected mice $(178.22 \pm 15.63 \mathrm{~s}$ vs. vehicle, $P<$ 0.01). However, implantation of PGA alone or hNPCs made no differences to these values compared with vehicle injection ( $n=15$ per group; Fig. $6 \mathrm{~b}$ ). These results indicated that hNPC-PGA transplantation promoted motosensory functional recovery without causing the mice to exhibit abnormal neurological behaviors after $\mathrm{HI}$ brain injury.

We assessed the capacity of implanted hNPC-PGA to improve hindlimb locomotor function, assessed by the open-filed BBB scores in the rats with SCI. In the ipsilateral lesioned side, hNPC-PGA implantation induced significant functional recovery at 14, 21, 28, 35, 42, 49, and 56 days post-transplantation compared with vehicle injection $(6.75 \pm 1.27$ vs. $2.55 \pm 0.84, P<0.01 ; 7.55 \pm 0.93$ vs. $3.86 \pm 0.66, P<0.01$; $9.34 \pm 1.25$ vs. $4.08 \pm 1.20, P<$ $0.01 ; 9.50 \pm 1.22$ vs. $4.79 \pm 1.24, P<0.01 ; 9.57 \pm 1.22$ vs. $4.92 \pm 1.41, P<0.05 ; 9.41 \pm 0.98$ vs. $5.19 \pm 1.18, p<0.05$; and $9.45 \pm 1.23$ vs. $5.38 \pm 1.35, P<0.05$, respectively; $n=$ 15 per group; Fig. $6 \mathrm{c}$ ). The hemisection of the spinal cord led to severance of many blood vessels and may have caused hypoxia and/or ischemia-associated secondary injury on the unlesioned contralateral side ${ }^{18}$. Hence, both hindlimbs were damaged. The performance of the contralateral side appeared to be similar to that of the ipsilateral side. hNPC-PGA implantation significantly increased locomotion of contralateral hindlimb at 14, 42, and 56 days post-transplantation compared with vehicle injection $(9.70 \pm 0.98$ vs. $6.38 \pm 0.80, P<0.05 ; 11.54 \pm 1.05$ vs. $8.99 \pm 1.06, P<0.05$; and $11.72 \pm 1.05$ vs. $9.08 \pm 0.82$, $P<0.05$, respectively; Fig. 6d). However, rats implanted with PGA alone or hNPCs ( $n=15$ per group) showed no differences in the locomotor functions of either hindlimb compared with the vehicle-injected rats (Fig. 6d). These data demonstrated that hNPC-PGA transplantation improved hindlimb locomotor performance without causing the rat to show abnormal neurological behaviors after SCI. We also evaluated the effects of hNPC-PGA implantation on postinjury development of sensory deficits associated with neuropathic pain in SCI, mechanical allodynia via the von Frey test. Compared with a day before the induction of hemisection SCI, the mechanical withdrawal threshold in vehicle-injected rats was markedly decreased on ipsilateral lesion side at $14,28,42$, and 56 days post-injection $(2.20 \pm 0.79,1.80 \pm 0.70,1.55 \pm 0.52$, $1.57 \pm 0.74$, respectively; $n=15$; Fig. 6e). These data indicate the development of mechanical allodynia persisting until 56 days after SCI. Rats implanted with PGA alone or hNPCs ( $n=15$ per group) showed no difference in the mechanical withdrawal threshold on ipsilateral lesion side compared with the vehicle-injected rats. However, the decrease of mechanical withdrawal threshold was significantly less in hNPC-PGA implanted rats at $14,28,42$, and 56 days post-transplantation compared with vehicle injection $(6.00 \pm 0.94$ vs. vehicle, $P<0.05$; $5.20 \pm 0.87$ vs. vehicle, $P<0.05 ; 5.01 \pm 0.69$ vs. vehicle, $P<$ 0.05 ; and $5.52 \pm 0.86$ vs. vehicle, $P<0.05$, respectively; $n=$ 15 per group; Fig. 6e). Hence, hNPC-PGA complex transplantation attenuated mechanical allodynia after SCI. 


\section{Discussion}

HI brain injury in neonatal mouse might offer a permissive environment to reconstitute the injured tissue on its own because young developing brain would even offer a receptive environment for grafting multipotent NPCs than the adult spinal cord. But this is not the case. It should be noted that neonatal HI brain injury is far beyond the period of cortical neurogenesis, which ends in fetal life. In this study, the postnatal mouse HI brain injury, a well-established model of sever HI encephalopathy or cerebral palsy in human infants, leads to extensive tissue loss (a large cystic infarction cavity occupying a significant portion of the cerebral hemisphere with its attendant glial scar and inflammation) ${ }^{35}$ due to loss of trophic factors, blood supply and synaptic input from neighboring cells, and widespread disturbances in maturation of neuronal or glial cells, resulting in altered neuronal connections and behavioral disabilities ${ }^{36,37}$. In this challenging environment, all neural cell types have been severely damaged, their interactions distorted and disconnected, and glial support disrupted. Thus, the requirements for repairing tissue lost to $\mathrm{HI}$ brain injury are so demanding that current stem cell transplantation strategies has only shown modest structural and functional benefits. Even the most "capable" multipotent NPCs need intrinsic organization, blood supply and a template to guide neural regeneration within the huge cystic infarction cavity. Like HI brain injury, repair of the injured spinal cord has been a major challenge for neuroscientists due to the inhibitory milieu of the adult CNS. The patterns of SCI are remarkably heterogeneous; the regional severity of cord injuries can differ both segmentally and longitudinally, and it mostly affects both central gray matter and the ascending and descending white matter tracts. Thus, cell or stem cell-based therapy for severe SCI with complete loss of function below the injured segmental levels would not be effective if confined simply to the delivery of some types of neurons and/or glia $^{38}$.

In the present study, we demonstrated that clinical applicable, primary hNPC-PGA scaffold grafts into the infarction cavity of neonatal HI brain injury and hemisection cavity of the adult spinal cord promoted neural regeneration and motorsensory functional improvement and helped to rebuild some damaged neuronal circuits. The interactions between not only hNPCs and scaffold but also the hNPC-scaffold complexes and the host brain and spinal cord tissue appeared to induce specific regenerative process and cellular responses, including survival, migration and differentiation of donor-derived cells, neurite outgrowth and axonal extension over long distances, reduction of brain infarction and spinal cord lesion volume, inhibition of glial scar formation and microglia/macrophage infiltration, polarization change of microglia/macrophage from M1 to M2 type, and neovascularization. Implanted cells seeded onto scaffold migrated and engrafted well into the lesion center and penumbra, and they differentiated into neurons and glia, although most of them remained immature. The differentiation patterns of donor-derived cells seeded on PGA scaffold in in vivo studies are a little different between neonatal HI brain injury and adult SCI. However, the majority of grafted cells in both models still remain immature state. Hence, although implanted hNPCs seeded on PGA scaffold appear to be capable of repopulating damaged brain and spinal cord regions, the vast volume of tissue and cell loss and inhibitory milieu of the CNS would seem to preclude differentiation of the grafted cells and even partial cell replacement.

Although implanted hNPCs showed a prolonged survival in rodent brain ${ }^{39-41}$, recent studies demonstrate that the immune response of neonatal mouse brains is uniquely sensitive against xenografts derived from human fetal brain ${ }^{42,43}$ and grafted cells rarely observed at 5 weeks or later post-transplantation ${ }^{43,44}$. In this study, hNPCs implanted into HI-injured brain have rarely been observed more than 2 months after transplantation. Implanted hNPCs appear not to survive well for a long time in highly inflammatory, inhospitable HI-injured brain following xenotransplantation despite of the use of immunosuppressant. A comprehensive knowledge of how transplanted hNPCs exert their therapeutic effects in CNS injury is still lacking and alternative pathways of hNPCsmediated repair should also be considered. Neuronal differentiation of implanted NPCs could promote restoration of disrupted circuitry by formation of bridges or bypass connections ${ }^{45}$ or may provide trophic support, enhancing neuroprotection and regeneration ${ }^{44,46}$. Additionally, accumulating evidences suggest that transplanted NPCs can have a variety of effects on the host microenvironment. NPCs, especially undifferentiated cells, release anti-inflammatory or immunomodulatory molecules at the site of tissue damage, and, in turn, promote functional recovery from CNS injuries ${ }^{47,48}$. Stem cell transplantation promotes regeneration of host CNS via favorable changes in the cytokine and growth factor milieu in the damaged CNS, thereby stimulating endogenous repair system, regardless of prolonged survival of grafted cells ${ }^{44,49-51}$. Hence, in this study, neurobehavioral recovery up to 11 weeks after transplantation of hNPCs into HI-injured brain may indicate that the limited presence of hNPCs implanted into mouse brain could ameliorate neurodegenerative environment through not functional integration of donor-derived cells but activating endogenous neurorestorative mechanisms that include neurogenesis, neural plasticity, angiogenesis, and anti-inflammation. 
Taken together, in this study, hNPC-scaffold complex grafts significantly restored motosensory function in brain injury and SCI, and attenuated neuropathic pain in SCI. Therefore, the structural and functional benefits following hNPC-PGA complex grafts in brain injury and SCI might be induced through multimodal actions of neuroprotection, neural plasticity, anti-inflammation/immunomodulation, and angiogenesis that limit secondary damage, and enhance neurite outgrowth and axonal extension over long distances, rather than by direct cell replacement or synaptic connections between grafted cells and host neurons. However, further researches are required to find out the difference in the repair mechanisms of hNPC-PGA grafts in neonatal HI brain injury and adult SCI.

The implantation procedure did not result in any adverse effects, such as infection, hemorrhage, tumor, atypical locomotion, aberrant motor coordination, or neurological deterioration. Regarding mortality following implantation, all animal groups in this study exhibited similar death rate (for mice with a HI brain injury, hNPCPGA, $n=5 / 55$; PGA, $n=6 / 55$; hNPCs, $n=6 / 55$; and vehicle, $n=4 / 55$, where $n=$ number of mice dead/total mice per group; for rats with SCI, hNPC-PGA, $n=7 / 60$; PGA, $n=7 / 60$; hNPCs, $n=8 / 60$; and vehicle, $n=6 / 60$, where $n=$ number of rats dead/total rats per group). These results suggest that the combination of hNPCs and biomaterial scaffolds contribute to the development of a multidisciplinary therapeutic approach for neonatal HI brain and adult SCI. Furthermore, the development of novel biomaterials that can enable greater control over biomaterial-stem cell interactions and targeted delivery of therapeutic proteins or drugs in combination with stem cells similarly engineered to express complementary factors or receptors might lead to a significant regeneration of extensively damaged tissue in the $\mathrm{CNS}^{9,16}$.

As natural or synthetic polymer-based scaffolds are proposed to serve as a delivery vehicle for stem cells and to connect the gap of the large lesions as contact guidance, NPCs combined with biomaterial scaffolds appear to favorably modify the microenvironment at the CNS injury site, structurally integrate with host tissue, and result in functional recovery ${ }^{9,16,18,52-54}$. Polymer scaffolds are fabricated to mimic some characteristics of natural ECM, which provides cells with structural and functional support. The individual components of ECM bind to surface receptors found on cells that can regulate cell behaviors, such as attachment, growth, migration, differentiation, survival, and other functions ${ }^{55-58}$. In addition, biodegradable polymers allow the diffusion of nutrients and metabolites, hinder the formation of scar tissue, concentrate neurotrophic growth and angiogenic factors, facilitate neurite outgrowth and axonal extension, and aid in connecting the neural network lost in the injury $^{12,18,20,59-62}$. Thus, the combinatorial approach of biomaterial, stem cells and biomolecules might change the local signals in the microenvironment of a brain injury or SCI, promoting the repair of injured CNS tissue with improved neurological function. However, a previous study showed that implantation of PLGA scaffold alone with specified architecture tailored to enhance spinal cord repair substantially improved function in SCI model ${ }^{18}$. By contrast, implantation of PGA scaffold in this study and PLGA scaffold designed to maintain stemness of mesenchymal stem cells (MSCs) was not sufficiently efficacious for brain injury and SCI recovery ${ }^{63}$. These data suggest that the appropriate design and fabrication of synthetic scaffold is crucial for actualization of functional biological effects. The therapeutic potential of biologic ECM scaffolds derived from mammalian tissue, both soluble and insoluble, for CNS repair has only recently been recognized ${ }^{64}$. Hence, further work is required to evaluate the response of neural tissue to biologic ECM scaffold materials in various CNS injury models.

Many experiments have been performed in vitro to evaluate the efficacy of scaffolds for NPC growth and differentiation ${ }^{16-20}$ however, whether the conditions that enable optimal and long term cell culture can be directly translated to in vivo experiment, as well as other important factors, including the optimal time window for transplantation of the NPC-scaffold complex into the injured brain, remains unknown. Nevertheless, some recent studies showed the considerable potential of grafted NPCs combined with biomaterial scaffolds in brain injury. In an adult ischemic brain injury model, implanted rat fetal NPCs within a collagen hydrogel promoted tissue repair better than NPCs alone ${ }^{65}$, and implanted ESCderived hNPCs with Matrigel ${ }^{\mathrm{TM}}$ significantly decreased infarct volume and increased sensorimotor and cognitive function $^{66}$. In neonatal HI brain injury, immortalized mouse NPCs-PGA complex implanted into the infarction cavity showed robust engraftment and differentiation of donor-derived cells, decreased parenchymal loss and the extent of inflammation and glial scarring, and increased neurite arborization of host and donor-derived neurons ${ }^{9}$. More recently, NPCs seeded onto PLGA microparticles implanted into an adult stroke model confirmed that cells integrated efficiently within the host tissue and formed primitive neural tissue ${ }^{67}$. Additionally, vascular endothelial growth factor (VEGF)-loaded PLGA microparticles with immortalized hNPCs were transplanted into an adult stroke model. The data showed that significant endothelial infiltration and neovascularization within the damaged hemisphere, but neither the survival nor the differentiation of donor-derived cells was enhanced ${ }^{68}$.

For SCI repair, both NPCs and MSCs using a variety of biomaterial scaffolds (PLGA scaffolds or conduit, PLGA film, poly[ $\varepsilon$-caprolactone] scaffold, self-assembling 
peptide nanofiber scaffold, collagen Gelfoam, chitosan channels, gelatin sponge, HPMA-RGD hydrogel, etc.) have been used ${ }^{15}$. Although implanted NPCs and MSCs seeded within polymer scaffolds have different mechanisms in post-SCI functional recovery, varying degree of axonal regeneration, behavioral improvement, survival and differentiation of donor-derived cells, reduced inflammatory response and scar formation, and angiogenesis have been reported. Recently, implantation of human MSCs (hMSCs) seeded on unique PLGA scaffold that augmented stemness, engraftment, and function of hMSCs demonstrated motosensory improvement, pain and tissue damage mitigation, and myelin preservation in hemisection SCI. These scaffolded hMSCs supported the propriospinal projections, neuromuscular junctions, and reticulospinal reinnervation to activate central pattern generator for restoring locomotion via multiple mechanisms_-neurotrophism, neurogenesis, angiogenesis, antiautoimmunity, and antiinflammation ${ }^{63}$. Such an understanding of these uncovered neural circuits and their mechanisms will undoubtedly help inform the development of rehabilitation therapies after SCI. In some other recent studies, NPCs were also reported to have the potential to reconstitute lesion sites with neurons and glia in $\mathrm{SCI}^{53,69,70}$. Furthermore, homologous reconstitution of the lesioned adult spinal cord with caudalized NPCs or with spinal cord-derived NPCs supported the robust regeneration of corticospinal axons ${ }^{71}$. Genetically modified NPCs combined with biomaterial scaffolds implanted into the SCI site increased the survival and neural differentiation of donor-derived cells as well as both behavioral and electrophysiological recovery ${ }^{72,73}$. Furthermore, newly developed injectable and gel-like three-dimensional electrospun nanofibers fostering a highly favorable environment for hNPC stemness and viability will broaden their applicability for brain injury and $\mathrm{SCI}^{19}$. These studies represent a substantial step forward in the technological development for clinical translation of this approach.

In this study, hNPC-scaffold grafts appear to reform the transcallosal connections between donor-derived or hostderived neurons and their targets in both cerebral hemispheres in HI brain injury and protect some injured corticospinal fibers in SCI. These histological and axonal tract tracing findings help to explain possible mechanisms leading to the motosensory recovery after brain injury and SCI. However, the mechanisms by which hNPC-PGA complex facilitate functional recovery including regeneration of propriospinal, raphespinal or reticulospinal projections, and neuromuscular junctions clearly require further investigation. Many more studies need to be conducted to increase the number of regenerating axons, achieve the proper directionality of the ascending and descending propriospinal tracts in $\mathrm{SCI}$, and optimize trophic and angiogenic support to promote neurite extension and axonal growth. In addition, electrophysiological recordings showing efficient axonal connectivity should be performed to analyze functional recovery.

The formation of a dense glial scar composed of astrocytes, oligodendrocytes, myelin, cellular debris, and microglia/macrophage at the injury site is one of the major challenges for long-distance axonal regeneration in the $\mathrm{CNS}^{5,6,16}$. A recent study revealed that astrocyte scar formation aids rather than prevents CNS axon regenera$\operatorname{tion}^{74}$. However, the abundance of other data shows that reactive astrocytes seem to be a barrier to severed axons. In addition, inflammatory cells, including highly activated microglia/macrophages that are present within the lesion center and penumbra, induce extensive axonal retraction $^{75}$. Hence, it appears that a decrease in astrocyte and microglia/macrophage infiltration, and a change of polarization of microglia/macrophage from M1 to M2 type following injury would be favorable for axon regrowth. Although the mechanism by which hNPC-PGA augments motosensory recovery is not yet known, hNPC-PGA complex or PGA scaffold appears to inhibit the ingrowth of reactive astrocytes and microglia/ macrophages into the CNS injury site, as well as the ingrowth of other various cell types that may be associated with inflammation and glial scar formation ${ }^{9,15,18,59}$. This anti-inflammatory effect of the hNPC-PGA complex or PGA scaffold through inhibiting astrocyte and microglia/ macrophage infiltration, and the polarization change of microglia/macrophage into anti-inflammatory activation following CNS injury may partly contribute to the reduction of tissue loss from secondary injury processes as well as the promotion of axonal growth. The hNPCs used in this study generated not only neurotrophins (BDNF [brain-derived neurotrophic factor], NTF3 [neurotrophin3], NTF4 [neurotrophin-4], and NGF [nerve growth factor]) but also other trophic and angiogenic factors, including VEGF, FGF2, and GDNF (glial cell line-derived neurotrophic factor). Furthermore, hNPC transplantation markedly attenuates astrogliosis, microgliosis, and the expression of pro-inflammatory mediators, as well as modulating immune responses through interactions of the implanted NPCs with T cells, microglia, or dendritic cells in a variety of neurological disease models ${ }^{23,39,76,77}$. In particular, we also displayed that hNPCs attenuated microglial activation through cell-to-cell contact and secretory molecules (TGF [transforming growth factor)$\beta 1$, IL4 [interleukin-4], IL13 [interleukin-13], CX3CL1, $\mathrm{CD} 200$, and $\mathrm{CD} 47]^{23}$. These findings suggest that not only PGA scaffold but also the hNPCs appear to prevent the formation of scar tissue and inflammatory reactions and concentrate neurotrophic and angiogenic factors, which contribute to the reduction of parenchymal loss, 
induction of survival and differentiation of donor-derived cells, and promotion of neurite outgrowth and axonal extension.

Therefore, future investigations might involve the development of smart biomaterials which have mechanical properties equivalent to those of CNS tissues, appropriate ligand presentation for implantation of different stem cell types, proper trophic factor sequestering ability, or capability to enhance tissue reconstruction and modulate inflammatory response. In combination with hNPCs genetically or non-genetically engineered to express disease-modifying molecules, this will provide a versatile tool for the treatment of brain injury and SCI.

\section{Acknowledgements}

This study was supported by grants from National Research Foundation (NRF; 2013M3A9B4076545) and Korean Health Technology R\&D Project (HI14C1564, HI16C1089). The authors thank Yonsei-Carl Zeiss Advanced Imaging Center, Yonsei University College of Medicine, for their technical assistance.

\begin{abstract}
Author details
'Department of Pediatrics, Severance Children's Hospital, Yonsei University College of Medicine, Seoul 03722, Korea. ${ }^{2}$ Brain Korea 21 Plus Project for Medical Science, Yonsei University College of Medicine, Seoul 03722, Korea. ${ }^{3}$ Yonsei Biomedical Research Institute, Yonsei University College of Medicine, Seoul 03722, Korea. ${ }^{4}$ Department of Physiology, Brain Research Institute, Epilepsy Research Institute, Yonsei University College of Medicine, Seoul 03722, Korea.
\end{abstract}

\section{Conflict of interest}

The authors declare that they have no conflict of interest.

\section{Publisher's note}

Springer Nature remains neutral with regard to jurisdictional claims in published maps and institutional affiliations

Supplementary information accompanies this paper at https://doi.org/ 10.1038/s12276-018-0054-9.

Received: 18 March 2017 Revised: 18 December 2017 Accepted: 21 December 2017.

Published online: 20 April 2018

\section{References}

1. Douglas-Escobar, M. \& Weiss, M. D. Hypoxic-ischemic encephalopathy: a review for the clinician. JAMA Pediatr. 169, 397-403 (2015).

2. Davidson, J. O., Wassink, G., van den Heuii, L. G., Bennet, L. \& Gunn, A. J. Therapeutic hypothermia for neonatal hypoxic-ischemic encephalopathywhere to from here? Front. Neurol. 6, 198 (2015).

3. Mokin, M., Rojas, H. \& Levy, E. I. Randomized trials of endovascular therapy for stroke-impact on stroke care. Nat. Rev. Neurol. 12, 86-94 (2016).

4. Fu, Y., Liu, Q., Anrather, J. \& Shi, F. D. Immune interventions in stroke. Nat. Rev. Neurol. 11, 524-535 (2015).

5. Bradbury, E. J. \& McMahon, S. B. Spinal cord repair strategies: why do they work? Nat. Rev. Neurosci. 7, 644-653 (2006).

6. Thuret, S., Moon, L. D. \& Gage, F. H. Therapeutic interventions after spinal cord injury. Nat. Rev. Neurosci. 7, 628-643 (2006).

7. Gage, F. H. \& Temple, S. Neural stem cells: generating and regenerating the brain. Neuron 80, 588-601 (2013).

8. Daadi, M. M. et al. Human neural stem cell grafts modify microglial response and enhance axonal sprouting in neonatal hypoxic-ischemic brain injury. Stroke 41, 516-523 (2010).
9. Park, K. I., Teng, Y. D. \& Snyder, E. Y. The injured brain interacts reciprocally with neural stem cells supported by scaffolds to reconstitute lost tissue. Nat. Biotechnol. 20, 1111-1117 (2002).

10. Imitola, J. et al. Directed migration of neural stem cells to sites of CNS injury by the stromal cell-derived factor 1alpha/CXC chemokine receptor 4 pathway. Proc. Natl. Acad. Sci. USA 101, 18117-18122 (2004).

11. Park, K. I. et al. Acute injury directs the migration, proliferation, and differentiation of solid organ stem cells: evidence from the effect of hypoxiaischemia in the CNS on clonal "reporter" neural stem cells. Exp. Neurol. 199, 156-178 (2006).

12. Park, K. I. et al. Neural stem cells may be uniquely suited for combined gene therapy and cell replacement: Evidence from engraftment of Neurotrophin-3expressing stem cells in hypoxic-ischemic brain injury. Exp. Neurol. 199, 179-190 (2006).

13. Lindvall, O. \& Kokaia, Z. Stem cells in human neurodegenerative disorderstime for clinical translation? J. Clin. Invest. 120, 29-40 (2010).

14. Barnabe-Heider, F. \& Frisen, J. Stem cells for spinal cord repair. Cell Stem Cell 3, 16-24 (2008).

15. Shrestha, B. et al. Repair of injured spinal cord using biomaterial scaffolds and stem cells. Stem Cell Res. Ther. 5, 91 (2014).

16. Orive, G., Anitua, E., Pedraz, J. L. \& Emerich, D. F. Biomaterials for promoting brain protection, repair and regeneration. Nat. Rev. Neurosci. 10, 682-692 (2009).

17. Hofstetter, C. P. et al. Allodynia limits the usefulness of intraspinal neural stem cell grafts; directed differentiation improves outcome. Nat. Neurosci. 8, 346-353 (2005).

18. Teng, Y. D. et al. Functional recovery following traumatic spinal cord injury mediated by a unique polymer scaffold seeded with neural stem cells. Proc. Natl. Acad. Sci. USA 99, 3024-3029 (2002).

19. Lee, S., Yun, S., Park, K. I. \& Jang, J. H. Sliding fibers: slidable, injectable, and gellike electrospun nanofibers as versatile cell carriers. ACS Nano 10, 3282-3294 (2016).

20. Skop, N. B., Calderon, F., Cho, C. H., Gandhi, C. D. \& Levison, S. W. Improvements in biomaterial matrices for neural precursor cell transplantation. Mol. Cell Ther 2, 19 (2014).

21. Kim, H. T. et al. Human neurospheres derived from the fetal central nervous system are regionally and temporally specified but are not committed. Exp. Neurol. 199, 222-235 (2006).

22. Peng, W. et al. Systemic administration of an antagonist of the ATP-sensitive receptor P2X7 improves recovery after spinal cord injury. Proc. Natl. Acad. Sci. USA 106, 12489-12493 (2009).

23. Lee, I. S. et al. Human neural stem cells alleviate Alzheimer-like pathology in a mouse model. Mol. Neurodegener. 10, 38 (2015).

24. Basso, D. M., Beattie, M. S. \& Bresnahan, J. C. A sensitive and reliable locomotor rating scale for open field testing in rats. J. Neurotrauma 12, 1-21 (1995).

25. Dixon, W. J. Efficient analysis of experimental observations. Annu. Rev. Pharmacol. Toxicol. 20, 441-462 (1980).

26. Mooney, D. J. et al. Stabilized polyglycolic acid fibre-based tubes for tissue engineering. Biomaterials 17, 115-124 (1996).

27. Puelacher, W. C. et al. Design of nasoseptal cartilage replacements synthesized from biodegradable polymers and chondrocytes. Biomaterials 15, 774-778 (1994).

28. Kim, W. S. et al. Cartilage engineered in predetermined shapes employing cell transplantation on synthetic biodegradable polymers. Plast. Reconstr. Surg. 94, 233-237 (1994). discussion238-40.

29. Vannucci, R. C. Experimental models of perinatal hypoxic-ischemic brain damage. Apmis. Suppl. 40, 89-95 (1993).

30. Akesson, E. et al. Long-term culture and neuronal survival after intraspina transplantation of human spinal cord-derived neurospheres. Physiol. Behav. 92, 60-66 (2007)

31. Kriegstein, A. \& Alvarez-Buylla, A. The glial nature of embryonic and adult neural stem cells. Annu. Rev. Neurosci. 32, 149-184 (2009).

32. Silver, J. \& Miller, J. H. Regeneration beyond the glial scar. Nat. Rev. Neurosci. $\mathbf{5}$ 146-156 (2004).

33. Mosser, D. M. \& Edwards, J. P. Exploring the full spectrum of macrophage activation. Nat. Rev. Immunol. 8, 958-969 (2008).

34. David, S. \& Kroner, A. Repertoire of microglial and macrophage responses after spinal cord injury. Nat. Rev. Neurosci. 12, 388-399 (2011).

35. Fitch, M. T., Doller, C., Combs, C. K., Landreth, G. E. \& Silver, J. Cellular and molecular mechanisms of glial scarring and progressive cavitation: in vivo and 
in vitro analysis of inflammation-induced secondary injury after CNS trauma. J. Neurosci. 19, 8182-8198 (1999).

36. Stone, B. S. et al. Delayed neural network degeneration after neonatal hypoxiaischemia. Ann. Neurol. 64, 535-546 (2008).

37. Barrett, R. D. et al. Destruction and reconstruction: hypoxia and the developing brain. Birth Defects Res. 81, 163-176 (2007).

38. Goldman, S. A. Stem and progenitor cell-based therapy of the central nervous system: hopes, hype, and wishful thinking. Cell Stem Cell 18, 174-188 (2016)

39. Rota Nodari, L. et al. Long-term survival of human neural stem cells in the ischemic rat brain upon transient immunosuppression. PLoS One 5, e14035 (2010).

40. Kelly, S. et al. Transplanted human fetal neural stem cells survive, migrate, and differentiate in ischemic rat cerebral cortex. Proc. Natl. Acad. Sci. USA 101, 11839-11844 (2004).

41. Windrem, M. S. et al. Neonatal chimerization with human glial progenitor cells can both remyelinate and rescue the otherwise lethally hypomyelinated shiverer mouse. Cell Stem Cell 2, 553-565 (2008).

42. Mattis, V. B. et al. Neonatal immune-tolerance in mice does not prevent xenograft rejection. Exp. Neurol. 254, 90-98 (2014).

43. Janowski, $M$. et al. Neonatal desensitization does not universally prevent xenograft rejection. Nat. Methods 9, 856-858 (2012).

44. Lee, I. S. et al. Neurogenin-2-transduced human neural progenitor cells attenuate neonatalhypoxic-ischemic brain injury. Transl. Res. 183, 121-136 (2017).

45. Abematsu, M. et al. Neurons derived from transplanted neural stem cells restore disrupted neuronal circuitry in a mouse model of spinal cord injury. J. Clin. Invest. 120, 3255-3266 (2010).

46. Yan, J. et al. Extensive neuronal differentiation of human neural stem cell grafts in adult rat spinal cord. PLoS Med. 4, e39 (2007).

47. Bacigaluppi, M. et al. Delayed post-ischaemic neuroprotection following systemic neural stem cell transplantation involves multiple mechanisms. Brain 132, 2239-2251 (2009).

48. Pluchino, S. et al. Injection of adult neurospheres induces recovery in a chronic model of multiple sclerosis. Nature 422, 688-694 (2003).

49. van Velthoven, C. T., Kavelaars, A., van Bel, F. \& Heijnen, C. J. Repeated mesenchymal stem cell treatment after neonatal hypoxia-ischemia has distinct effects on formation and maturation of new neurons and oligodendrocytes leading to restoration of damage, corticospinal motor tract activity, and sensorimotor function. J. Neurosci. 30, 9603-9611 (2010).

50. van Velthoven, C. T., Kavelaars, A., van Bel, F. \& Heijnen, C. J. Mesenchymal stem cell transplantation changes the gene expression profile of the neonatal ischemic brain. Brain Behav. Immun. 25, 1342-1348 (2011).

51. Chen, L. et al. Human neural precursor cells promote neurologic recovery in a viral model of multiple sclerosis. Stem Cell Rep. 2, 825-837 (2014).

52. Huang, Y. C. \& Huang, Y. Y. Biomaterials and strategies for nerve regeneration. Artif. Organs 30, 514-522 (2006).

53. Lu, P. et al. Long-distance growth and connectivity of neural stem cells after severe spinal cord injury. Cell 150, 1264-1273 (2012).

54. Zeng, X. et al. Bone marrow mesenchymal stem cells in a three-dimensional gelatin sponge scaffold attenuate inflammation, promote angiogenesis, and reduce cavity formation in experimental spinal cord injury. Cell Transplant. 20, 1881-1899 (2011).

55. Olson, H. E. et al. Neural stem cell- and Schwann cell-loaded biodegradable polymer scaffolds support axonal regeneration in the transected spinal cord. Tissue Eng. Part A 15, 1797-1805 (2009).
56. Cholas, R. H., Hsu, H. P. \& Spector, M. The reparative response to cross-linked collagen-based scaffolds in a rat spinal cord gap model. Biomaterials $\mathbf{3 3}$ 2050-2059 (2012)

57. Hejcl, A. et al. HPMA-RGD hydrogels seeded with mesenchymal stem cells improve functional outcome in chronic spinal cord injury. Stem Cells Dev. 19, 1535-1546 (2010).

58. Lee, K. Y. \& Mooney, D. J. Cell-interactive polymers for tissue engineering. Fibers Polym. 2, 51-57 (2001).

59. Straley, K. S., Foo, C. W. \& Heilshorn, S. C. Biomaterial design strategies for the treatment of spinal cord injuries. J. Neurotrauma 27, 1-19 (2010).

60. Nomura, H., Tator, C. H. \& Shoichet, M. S. Bioengineered strategies for spinal cord repair. J. Neurotrauma 23, 496-507 (2006).

61. Piantino, J., Burdick, J. A., Goldberg, D., Langer, R. \& Benowitz, L. I. An injectable, biodegradable hydrogel for trophic factor delivery enhances axonal rewiring and improves performance after spinal cord injury. Exp. Neurol. 201, 359-367 (2006).

62. Tate, C. C. et al. Laminin and fibronectin scaffolds enhance neural stem cell transplantation into the injured brain. J. Tissue Eng. Regen. Med. 3, 208-217 (2009).

63. Ropper, A. E. et al. Defining recovery neurobiology of injured spinal cord by synthetic matrix-assisted hMSC implantation. Proc. Natl. Acad. Sci. USA 114 E820-E829 (2017).

64. Meng, F., Modo, M. \& Badylak, S. F. Biologic scaffold for CNS repair. Regen. Med. 9, 367-383 (2014).

65. Yu, H. et al. Combinated transplantation of neural stem cells and collagen type I promote functional recovery after cerebral ischemia in rats. Anat. Rec. (Hoboken). 293, 911-917 (2010).

66. Jin, K. et al. Transplantation of human neural precursor cells in Matrigel scaffolding improves outcome from focal cerebral ischemia after delayed postischemic treatment in rats. J. Cereb. Blood Flow. Metab. 30, 534-544 (2010).

67. Bible, E. et al. The support of neural stem cells transplanted into strokeinduced brain cavities by PLGA particles. Biomaterials 30, 2985-2894 (2009).

68. Bible, E. et al. Neo-vascularization of the stroke cavity by implantation of human neural stem cells on VEGF-releasing PLGA microparticles. Biomaterials 33, 7435-7446 (2012)

69. Bonner, J. F. et al. Grafted neural progenitors integrate and restore synaptic connectivity across the injured spinal cord. J. Neurosci. 31, 4675-4686 (2011).

70. Lu, P. et al. Long-distance axonal growth from human induced pluripotent stem cells after spinal cord injury. Neuron 83, 789-796 (2014).

71. Kadoya, K. et al. Spinal cord reconstitution with homologous neural grafts enables robust corticospinal regeneration. Nat. Med. 22, 479-487 (2016).

72. Hwang, D. H. et al. Combination of multifaceted strategies to maximize the therapeutic benefits of neural stem cell transplantation for spinal cord repair. Cell Transplant. 20, 1361-1379 (2011)

73. Kim, B. G. et al. Implantation of polymer scaffolds seeded with neural stem cells in a canine spinal cord injury model. Cytotherapy 12, 841-845 (2010).

74. Anderson, M. A. et al. Astrocyte scar formation aids central nervous system axon regeneration. Nature 532, 195-200 (2016).

75. Silver, J. The glial scar is more than just astrocytes. Exp. Neurol. 286, 147-149 (2016).

76. Pluchino, S. et al. Neurosphere-derived multipotent precursors promote neuroprotection by an immunomodulatory mechanism. Nature 436, 266-271 (2005).

77. Cusimano, M. et al. Transplanted neural stem/precursor cells instruct phagocytes and reduce secondary tissue damage in the injured spinal cord. Brain 135, 447-460 (2012). 